\title{
New 4-Spiroannulated Tetrahydroisoquinolines by One-Pot Sequential Procedure. Isolation and Characterization of $\sigma$-Alkylpalladium Heck
}

\section{Intermediates}

Egle M. Beccalli, ${ }^{\star}$ Gianluigi Broggini, ${ }^{\S}$ Michela Martinelli, ${ }^{\star}$ Norberto Masciocchi, ${ }^{\S}$ Silvia Sottocornola

‡Istituto di Chimica Organica “A. Marchesini”, Facoltà di Farmacia, Università di Milano, via Venezian 21, 20133 Milano, Italy

${ }^{\S}$ Dipartimento di Scienze Chimiche e Ambientali, Università dell’ Insubria, via Valleggio 11, 22100 Como, Italy

gianluigi.broggini@uninsubria.it

\section{Supporting Information}




\section{General}

Melting points were measured with a Büchi B-540 heating unit and are uncorrected. IR spectra were recorded on a FT-IR spectrophotometer. Mass spectra were determined on an HPLC-MS LCQAdvantage Thermo Finnigan instrument. ${ }^{1} \mathrm{H}$ NMR and ${ }^{13} \mathrm{C}$ NMR spectra were recorded at 400 and 100 $\mathrm{MHz}$ on an AVANCE 400 Bruker. Elemental analyses were executed on Perkin-Elmer CHN Analyzer Series II 2400. Chemical shifts ( $\delta$ ) are given as ppm relative to the residual solvent peak (chloroform- $d I$ $7.25 \mathrm{ppm} / 77 \mathrm{ppm}$ ). Column chromatography was performed on a Merck silica gel 60, (mesh size 63$200 \mu \mathrm{m})$.

\section{General one-pot procedure for the preparation of spiro[isoquinoline-4,5'-isoxazole]}

\section{4a-d.}

A solution of $2(1 \mathrm{mmol})$ and TEA $(0,17 \mathrm{ml}, 1.2 \mathrm{mmol})$ in DMF $(3 \mathrm{ml})$ was dropped in a stirred solution of 1 (309 mg, $1 \mathrm{mmol}$ ) in DMF (3 mL). The resulting solution was heated at $60^{\circ} \mathrm{C}$ for $45 \mathrm{~min}$, then $\mathrm{Pd}(\mathrm{OAc})_{2}(11 \mathrm{mg}, 0.05 \mathrm{~mol} \%), \mathrm{PPh}_{3}(52 \mathrm{mg}, 0.20 \mathrm{~mol} \%)$ and $\mathrm{Cs}_{2} \mathrm{CO}_{3}(650 \mathrm{mg}, 2 \mathrm{mmol})$ were added in sequence. The mixture was taken to $120^{\circ} \mathrm{C}$ for $6 \mathrm{~h}$. Finally, 3 (276 mg, 1,2 mmol) was added and the mixture was stirred at $120^{\circ} \mathrm{C}$ for $18 \mathrm{~h}$. After concentration of the solvent under reduced pressure, the crude brown residue was purified by flash chromatography (EtPe / AcOEt 15:1).

\section{3'-(3,5-Dichloro-2,4,6-trimethylphenyl)-2-methyl-1,2,3,4'-tetraydro-spiro[isoquinoline-4,5'-}

isoxazole] (4a) - Yield: 30\%. M.p. $169-170^{\circ} \mathrm{C}$ (diisopropyl ether). ${ }^{1} \mathrm{H}$ NMR $\left(400 \mathrm{MHz}, \mathrm{CDCl}_{3}\right) \delta: 2.40$ $(6 \mathrm{H}, \mathrm{s}), 2.53(3 \mathrm{H}, \mathrm{s}), 2.56(3 \mathrm{H}, \mathrm{s}), 2.84,2.94(2 \mathrm{H}, \mathrm{AB}$ system, $J=11.2 \mathrm{~Hz}), 3.29,3.45(2 \mathrm{H}, \mathrm{AB}$ system, $J=18.4 \mathrm{~Hz}), 3.53,3.81(2 \mathrm{H}, \mathrm{AB}$ system, $J=15.0 \mathrm{~Hz}), 7.09(1 \mathrm{H}, \mathrm{d}, J=7.5 \mathrm{~Hz}), 7.28(1 \mathrm{H}, \mathrm{dd}$, $J=7.3,7.5 \mathrm{~Hz}), 7.35(1 \mathrm{H}, \mathrm{dd}, J=7.3,7.6 \mathrm{~Hz}), 7.56(1 \mathrm{H}, \mathrm{d}, J=7.6 \mathrm{~Hz}) ;{ }^{13} \mathrm{C} \mathrm{NMR}\left(100 \mathrm{MHz}, \mathrm{CDCl}_{3}\right)$ $\delta: 18.6(\mathrm{q}), 19.4(\mathrm{q}), 46.6(\mathrm{q}), 54.1(\mathrm{t}), 58.2(\mathrm{t}), 63.3(\mathrm{t}), 85.7(\mathrm{~s}), 126.1(\mathrm{~d}), 126.4(\mathrm{~d}), 127.8(\mathrm{~d}), 128.3$ (d), 129.3 (s), 133.6 (s), 134.0 (s), 135.2 (s), 135.9 (s), 137.2 (s), 156.5 (s). MS: m/z 388 (M ${ }^{+}$). Anal. calcd for $\mathrm{C}_{21} \mathrm{H}_{22} \mathrm{Cl}_{2} \mathrm{~N}_{2} \mathrm{O}$ : C, 64.79; H, 5.70; N, 7.20. Found C, 64.90; H, 5.41; N, 6.93. 


\section{2-Allyl-3' -(3,5-dichloro-2,4,6-trimethylphenyl)-1,2,3,4'-tetraydro-spiro[isoquinoline-4,5’'}

isoxazole] (4b) - Yield: 32\%. M.p. $132-133{ }^{\circ} \mathrm{C}$ (diisopropyl ether). ${ }^{1} \mathrm{H}$ NMR (400 MHz, $\left.\mathrm{CDCl}_{3}\right) \delta$ : $2.39(6 \mathrm{H}, \mathrm{s}), 2.56(3 \mathrm{H}, \mathrm{s}), 2.71(1 \mathrm{H}, \mathrm{d}, J=11.1 \mathrm{~Hz}), 3.10-3.17(2 \mathrm{H}, \mathrm{m}), 3.25,3.46(2 \mathrm{H}, \mathrm{AB}$ system, $J=$ $18.3 \mathrm{~Hz}), 3.32-3.36(1 \mathrm{H}, \mathrm{m}), 3.61,3.99(2 \mathrm{H}, \mathrm{AB}$ system, $J=14.9 \mathrm{~Hz}), 5.27(1 \mathrm{H}, \mathrm{d}, J=10.2 \mathrm{~Hz}), 5.31$ $(1 \mathrm{H}, \mathrm{d}, J=17.3 \mathrm{~Hz}), 5.85-6.01(1 \mathrm{H}, \mathrm{m}), 7.10(1 \mathrm{H}, \mathrm{d}, J=7.6 \mathrm{~Hz}), 7.28(1 \mathrm{H}, \mathrm{dd}, J=7.7,7.8 \mathrm{~Hz}), 7.35$ $(1 \mathrm{H}, \mathrm{dd}, J=7.8,7.6 \mathrm{~Hz}), 7.57(1 \mathrm{H}, \mathrm{d}, J=7.7 \mathrm{~Hz}) ;{ }^{13} \mathrm{C} \mathrm{NMR}\left(100 \mathrm{MHz}, \mathrm{CDCl}_{3}\right) \delta: 18.6(\mathrm{q}), 19.4(\mathrm{q})$, $54.2(\mathrm{t}), 56.6(\mathrm{t}), 59.5(\mathrm{t}), 61.3(\mathrm{t}), 85.9(\mathrm{~s}), 85.9(\mathrm{~s}), 119.3(\mathrm{t}), 126.1(\mathrm{~d}), 126.5(\mathrm{~d}), 127.8(\mathrm{~d}), 128.3(\mathrm{~d})$, 129.4 (s), 133.6 (s), 134.0 (s), 135.0 (d), 135.4 (s), 135.9 (s), 137.8 (s), 156.7 (s). MS: m/z $414\left(\mathrm{M}^{+}\right)$. Anal. calcd for $\mathrm{C}_{23} \mathrm{H}_{24} \mathrm{Cl}_{2} \mathrm{~N}_{2} \mathrm{O}$ : C, 66.51; H, 5.82; N, 6.74. Found C, 66.34; H, 6.07; N, 6.91.

\section{2-Cyclohexyl-3' -(3,5-dichloro-2,4,6-trimethylphenyl)-1,2,3,4'-tetraydro-spiro[isoquinoline-4,5'-}

isoxazole] (4c) - Yield: $45 \%$. M.p. $177-178^{\circ} \mathrm{C}$ (diisopropyl ether) ${ }^{1} \mathrm{H}$ NMR $\left(400 \mathrm{MHz}, \mathrm{CDCl}_{3}\right) \delta: 1.03-$ $1.47(6 \mathrm{H}, \mathrm{m}), 1.65-2.25(4 \mathrm{H}, \mathrm{m}), 2.41(6 \mathrm{H}, \mathrm{s}), 2.50-2.57(1 \mathrm{H}, \mathrm{m}), 2.56(3 \mathrm{H}, \mathrm{s}), 2.82,3.14(2 \mathrm{H}, \mathrm{AB}$ system, $J=11.1 \mathrm{~Hz}), 3.22,3.53(2 \mathrm{H}$, AB system, $J=18.4 \mathrm{~Hz}), 3.85,3.92(2 \mathrm{H}$, AB system, $J=15.0)$, $7.09(1 \mathrm{H}, \mathrm{d}, J=7.5 \mathrm{~Hz}), 7.26(1 \mathrm{H}, \mathrm{dd}, J=7.4,7.7 \mathrm{~Hz}), 7.32(1 \mathrm{H}, \mathrm{dd}, J=7.4,7.5 \mathrm{~Hz}), 7.55(1 \mathrm{H}, \mathrm{d}, J=$ $7.7 \mathrm{~Hz}) ;{ }^{13} \mathrm{C}$ NMR (100 MHz, $\left.\mathrm{CDCl}_{3}\right) \delta: 18.6(\mathrm{q}), 19.4(\mathrm{q}), 26.1(\mathrm{t}), 26.3(\mathrm{t}), 26.7(\mathrm{t}), 28.1(\mathrm{t}), 30.4(\mathrm{t})$, $52.9(\mathrm{t}), 54.2(\mathrm{t}), 55.8(\mathrm{t}), 63.3(\mathrm{~d}), 86.5(\mathrm{~s}), 126.0(\mathrm{~d}), 126.6(\mathrm{~d}), 127.5(\mathrm{~d}), 128.0(\mathrm{~d}), 129.5(\mathrm{~s}), 133.6$ (s), 134.0 (s), 135.8 (s), 136.0 (s), 138.3 (s), 156.7 (s). MS: m/z $456\left(\mathrm{M}^{+}\right)$. Anal. calcd for $\mathrm{C}_{26} \mathrm{H}_{30} \mathrm{Cl}_{2} \mathrm{~N}_{2} \mathrm{O}$ : C, 68.27; H, 6.61; N, 6.12. Found C, 68.25; H, 6.79; N, 6.22.

\section{2-Cyclopentyl-3'-(3,5-dichloro-2,4,6-trimethylphenyl)-1,2,3,4'-tetraydro-spiro[isoquinoline-}

4,5'-isoxazole] (4d) - Yield: 41\%. M.p. $191-192^{\circ} \mathrm{C}$ (diisopropyl ether). ${ }^{1} \mathrm{H}$ NMR (400 MHz, $\left.\mathrm{CDCl}_{3}\right) \delta$ : 1.42-1.56 (2H, m), 1.57-1.78 (4H, m), 1.80-2.05 (2H, m), 2.41 (6H, s), $2.56(3 \mathrm{H}, \mathrm{s}), 2.82-2.90(1 \mathrm{H}, \mathrm{m})$, 2.73, $3.21(2 \mathrm{H}, \mathrm{AB}$ system, $J=11.3 \mathrm{~Hz}), 3.25,3.51(2 \mathrm{H}, \mathrm{AB}$ system, $J=18.4 \mathrm{~Hz}), 3.59,3.98(2 \mathrm{H}, \mathrm{AB}$ system, $J=14.9), 7.10(1 \mathrm{H}, \mathrm{d}, J=7.5 \mathrm{~Hz}), 7.27(1 \mathrm{H}, \mathrm{dd}, J=7.3,7.5 \mathrm{~Hz}), 7.34(1 \mathrm{H}, \mathrm{dd}, J=7.3,7.6$ $\mathrm{Hz}), 7.56(1 \mathrm{H}, \mathrm{d}, J=7.6 \mathrm{~Hz}) ;{ }^{13} \mathrm{C} \mathrm{NMR}\left(100 \mathrm{MHz}, \mathrm{CDCl}_{3}\right) \delta: 18.6(\mathrm{q}), 19.4(\mathrm{q}), 24.4(\mathrm{t}), 24.5(\mathrm{t}), 30.7$ $(\mathrm{t}), 31.1(\mathrm{t}), 54.2(\mathrm{t}), 55.7(\mathrm{t}), 59.0(\mathrm{t}), 66.8(\mathrm{~d}), 86.0(\mathrm{~s}), 126.0(\mathrm{~d}), 126.6(\mathrm{~d}), 127.7(\mathrm{~d}), 128.2(\mathrm{~d})$, 
129.4 (s), 133.6 (s), 134.0 (s), 135.7 (s), 135.9 (s), 137.9 (s), 156.7 (s). MS: m/z 442 (M ${ }^{+}$). Anal. calcd for $\mathrm{C}_{25} \mathrm{H}_{28} \mathrm{Cl}_{2} \mathrm{~N}_{2} \mathrm{O}$ : C, 67.72; H, 6.36; N, 6.32. Found C, 67.71; H, 6.57; N, 6.09.

\section{General procedure for the preparation of the 4-methylene-1,2,3,4-tetrahydro-}

\section{isoquinolines 6a-c from palladacycles 9a-c.}

A solution of 9a-c $(1 \mathrm{mmol})$ and TEA $(0,42 \mathrm{ml}, 3 \mathrm{mmol})$ in $\mathrm{CH}_{3} \mathrm{CN}(10 \mathrm{~mL})$ was refluxed for $48 \mathrm{~h}$. After removal of the solvent under reduced pressure, $\mathrm{H}_{2} \mathrm{O}(10 \mathrm{~mL})$ was added and the aqueous layer was extracted with $\mathrm{CH}_{2} \mathrm{Cl}_{2}(2 \times 10 \mathrm{ml})$ The combined organic layers were dried $\left(\mathrm{Na}_{2} \mathrm{SO}_{4}\right)$ and eluted through a silica gel column (EtPe / AcOEt 15:1).

2-Methyl-4-methylene-1,2,3,4-tetrahydro-isoquinoline (6a) - Yield: 38\%. Oil. ${ }^{1} \mathrm{H}$ NMR (400 $\left.\mathrm{MHz}, \mathrm{CDCl}_{3}\right) \delta: 2.46(3 \mathrm{H}, \mathrm{s}), 3.35(2 \mathrm{H}, \mathrm{s}), 3.68(2 \mathrm{H}, \mathrm{s}), 5.04(1 \mathrm{H}, \mathrm{s}), 5.64(1 \mathrm{H}, \mathrm{s}), 7.06-7.09(1 \mathrm{H}, \mathrm{m})$, 7.18-7.24 (2H, m), 7.69-7.72 (1H, m); $\left.{ }^{13} \mathrm{C} \mathrm{NMR} \mathrm{(100} \mathrm{MHz,} \mathrm{CDCl}_{3}\right) \delta: 45.6(\mathrm{q}), 59.0(\mathrm{t}), 60.7(\mathrm{t}), 108.1$ (t), 123.8 (d), 127.0 (d), 127.1 (d), 128.2 (d), 132.2 (s), 135.2 (s), 140.0 (s). MS: m/z 159 (M ${ }^{+}$). Anal. calcd for $\mathrm{C}_{11} \mathrm{H}_{13} \mathrm{~N}$ : C, 82.97; H, 8.23; N, 8.80. Found C, 82.79; H, 7.97; N, 8.54.

2-Allyl-4-methylene-1,2,3,4-tetrahydro-isoquinoline (6b) - Yield: 41\%. Oil. ${ }^{1} \mathrm{H}$ NMR (400 MHz,

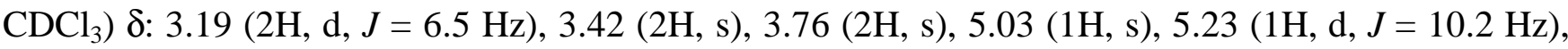
$5.27(1 \mathrm{H}, \mathrm{d}, J=17.2 \mathrm{~Hz}), 5.64(1 \mathrm{H}, \mathrm{s}), 5.95(1 \mathrm{H}, \mathrm{tdd}, J=6.5,10.2,17.2 \mathrm{~Hz}), 7.06-7.10(1 \mathrm{H}, \mathrm{m}), 7.19-$ $7.23(2 \mathrm{H}, \mathrm{m}), 7.68-7.72(1 \mathrm{H}, \mathrm{m}) ;{ }^{13} \mathrm{C}$ NMR $\left(100 \mathrm{MHz}, \mathrm{CDCl}_{3}\right) \delta: 56.8(\mathrm{t}), 58.4(\mathrm{t}), 60.7(\mathrm{t}), 108.4(\mathrm{t})$, 118.6 (t), 123.8 (d), 127.0 (d), 127.3 (d), 128.3 (d), 132.6 (s), 135.0 (s), 135.5 (d), 139.6 (s). MS: $m / z$ $185\left(\mathrm{M}^{+}\right)$. Anal. calcd for $\mathrm{C}_{13} \mathrm{H}_{15} \mathrm{~N}$ : C, 84.28; H, 8.16; N, 7.56. Found C, 84.24; H, 7.91; N, 7.29.

2-Cyclohexyl-4-methylene-1,2,3,4-tetrahydro-isoquinoline (6c) - Yield: 46\%. Oil. ${ }^{1} \mathrm{H}$ NMR (400 $\left.\mathrm{MHz}, \mathrm{CDCl}_{3}\right) \delta: 1.10-2.05(10 \mathrm{H}, \mathrm{m}), 2.47-2.53(1 \mathrm{H}, \mathrm{m}), 3.51(2 \mathrm{H}, \mathrm{s}), 3.89(2 \mathrm{H}, \mathrm{s}), 5.01(1 \mathrm{H}, \mathrm{s}), 5.60$ $(1 \mathrm{H}, \mathrm{s}), 7.07-7.11(1 \mathrm{H}, \mathrm{m}), 7.17-7.24(2 \mathrm{H}, \mathrm{m}), 7.66-7.71(1 \mathrm{H}, \mathrm{m}) ;{ }^{13} \mathrm{C} \mathrm{NMR}\left(100 \mathrm{MHz}, \mathrm{CDCl}_{3}\right) \delta: 26.2$ $(\mathrm{t}), 26.7(\mathrm{t}), 29.5(\mathrm{t}), 53.0(\mathrm{t}), 55.1(\mathrm{t}), 62.4(\mathrm{~d}), 107.7(\mathrm{t}), 123.9(\mathrm{~d}), 126.8(\mathrm{~d}), 127.3(\mathrm{~d}), 129.1(\mathrm{~d})$, 132.9 (s), 135.9 (s). MS: $m / z 227\left(\mathrm{M}^{+}\right)$. Anal. calcd for $\mathrm{C}_{16} \mathrm{H}_{21} \mathrm{~N}: \mathrm{C}, 84.53 ; \mathrm{H}, 9.31 ; \mathrm{N}, 6.16$. Found C, 84.24; H, 9.54; N, 6.19. 
General procedure for the preparation of the palladacycles 9a-c with stoichiometric

\section{amount of $\mathbf{P d}\left(\mathbf{P P h}_{3}\right)_{4}=$}

To a solution of 5a-c $(1 \mathrm{mmol})$ in $\mathrm{CH}_{3} \mathrm{CN}(10 \mathrm{~mL})$ were added $\mathrm{Pd}\left(\mathrm{PPh}_{3}\right)_{4}(1,15 \mathrm{~g}, 1 \mathrm{mmol})$ and TEA $(0,42 \mathrm{ml}, 3 \mathrm{mmol})$. The mixture was heated and stirred at reflux for $24 \mathrm{~h}$. After removal of the solvent under reduced pressure, $\mathrm{H}_{2} \mathrm{O}(10 \mathrm{~mL})$ was added and the aqueous layer was extracted with $\mathrm{CH}_{2} \mathrm{Cl}_{2}(2 \mathrm{x}$ $10 \mathrm{ml})$ The combined organic layers were dried $\left(\mathrm{Na}_{2} \mathrm{SO}_{4}\right)$ and eluted through a silica gel column (EtPe / AcOEt 7:3).

Compound 9a - Yield: 55\%. M.p. $128-130^{\circ} \mathrm{C}$ (diisopropyl ether). ${ }^{1} \mathrm{H}$ NMR $\left(400 \mathrm{MHz}, \mathrm{CDCl}_{3}\right) \delta$ : $1.56(1 \mathrm{H}, \mathrm{m}), 1.84(1 \mathrm{H}, \mathrm{dd}, J=3.0,9.2 \mathrm{~Hz}), 2.62(1 \mathrm{H}, \mathrm{m}), 2.71(1 \mathrm{H}, \mathrm{d}, J=10.2 \mathrm{~Hz}), 3.01(1 \mathrm{H}, \mathrm{d}, J=$ $10.2 \mathrm{~Hz}), 3.28(3 \mathrm{H}, \mathrm{d}, J=2.8 \mathrm{~Hz}), 3.62(1 \mathrm{H}, \mathrm{dd}, J=5.8,14.8 \mathrm{~Hz}), 5.11(1 \mathrm{H}, \mathrm{d}, J=14.8 \mathrm{~Hz}), 6.98(1 \mathrm{H}$, d, $J=7.3 \mathrm{~Hz}), 7.18-7.39(18 \mathrm{H}, \mathrm{m}) ;{ }^{13} \mathrm{C} \mathrm{NMR}\left(100 \mathrm{MHz}, \mathrm{CDCl}_{3}\right) \delta: 45.6(\mathrm{~d}), 50.7$ (t), $53.1(\mathrm{q}), 63.2(\mathrm{t})$, $65.3(\mathrm{t}), 126.7$ (d), 127.1 (d), 127.4 (d), 128.1 (d), 128.2 (d), 128.3 (d), 130.4 (d), 132.2 (s) 132.5 (s), $132.6(\mathrm{~s}), 135.0(\mathrm{~d}), 135.1(\mathrm{~d}), 140.2(\mathrm{~s}) . \mathrm{MS}: \mathrm{m} / z 655\left(\mathrm{M}^{+}\right)$calculated for the most abundant $\mathrm{Pd}$ isotope, ${ }^{106} \mathrm{Pd}$. Anal. calcd for $\mathrm{C}_{29} \mathrm{H}_{29} \mathrm{INPPd}$ : C, 53.11; H, 4.46; N, 2.14. Found C, 52.97; H, 4.76; N, 1.88.

Compound 9b - Yield: 66\%. M.p. $163-165^{\circ} \mathrm{C}$ (diisopropyl ether). ${ }^{1} \mathrm{H}$ NMR (400 MHz, $\left.\mathrm{CDCl}_{3}\right) \delta$ : $1.49-1.53(1 \mathrm{H}, \mathrm{m}), 1.83(1 \mathrm{H}, \mathrm{dd}, J=3.2, J=9.1 \mathrm{~Hz}), 2.43(1 \mathrm{H}, \mathrm{d}, J=10.4 \mathrm{~Hz}), 2.61-2.64(1 \mathrm{H}, \mathrm{m})$, 3.13-3.22 (1H, m), $3.23(1 \mathrm{H}, \mathrm{d}, J=10.4 \mathrm{~Hz}), 3.81(1 \mathrm{H}, \mathrm{dd}, J=5.5, J=14.7 \mathrm{~Hz}), 4.89(1 \mathrm{H}, \mathrm{dd}, J=2.8$, $J=9.9 \mathrm{~Hz}), 5.17(1 \mathrm{H}, \mathrm{d}, J=14.7 \mathrm{~Hz}), 5.43(1 \mathrm{H}, \mathrm{d}, J=17.3 \mathrm{~Hz}), 5.47(1 \mathrm{H}, \mathrm{d}, J=10.3 \mathrm{~Hz}), 6.65-6.71$ $(1 \mathrm{H}, \mathrm{m}), 6.98(1 \mathrm{H}, \mathrm{d}, J=7.4 \mathrm{~Hz}), 7.20-7.38(18 \mathrm{H}, \mathrm{m}),{ }^{13} \mathrm{C} \mathrm{NMR}\left(100 \mathrm{MHz}, \mathrm{CDCl}_{3}\right) \delta: 45.35(\mathrm{~d}), 50.41$ $(\mathrm{t}), 60.24(\mathrm{t}), 61.97(\mathrm{t}), 64.88(\mathrm{t}), 121.02(\mathrm{t}), 126.67(\mathrm{~d}), 127.21(\mathrm{~d}), 127.45(\mathrm{~d}), 128.07(\mathrm{~d}), 128.22(\mathrm{~d})$, 128.33 (d), 130.43 (d), 132.26 (s), 132.75 (s), 134.42 (d), 134.95 (d), 135.06 (d), 140.83 (s). MS: m/z $681\left(\mathrm{M}^{+}\right)$. Anal. calcd for $\mathrm{C}_{31} \mathrm{H}_{31} \mathrm{INPPd}$ : C, 54.60; H, 4.58; N, 2.05. Found C, 54.41; H, 4.81; N, 1.77 .

Compound 9c - Yield: 59\%. M.p. $158-160^{\circ} \mathrm{C}$ (diisopropyl ether). ${ }^{1} \mathrm{H}$ NMR $\left(400 \mathrm{MHz}, \mathrm{CDCl}_{3}\right) \delta: 1.10-$ $1.93(10 \mathrm{H}, \mathrm{m}), 2.13(1 \mathrm{H}, \mathrm{d}, J=11.5 \mathrm{~Hz}), 2.57(1 \mathrm{H}, \mathrm{d}, J=10.2 \mathrm{~Hz}), 2.65-2.68(1 \mathrm{H}, \mathrm{m}), 2.97(1 \mathrm{H}, \mathrm{d}, J=$ 
$10.2 \mathrm{~Hz}), 3.18-3.24(1 \mathrm{H}, \mathrm{m}), 3.93-4.00(1 \mathrm{H}, \mathrm{m}), 4.32(1 \mathrm{H}, \mathrm{dd}, J=6.2,14.6 \mathrm{~Hz}), 5.03(1 \mathrm{H}, \mathrm{d}, J=14.6$ $\mathrm{Hz}), 6.95(1 \mathrm{H}, \mathrm{d}, J=7.4 \mathrm{~Hz}), 7.15-7.39(18 \mathrm{H}, \mathrm{m}) ;{ }^{13} \mathrm{C} \mathrm{NMR}\left(100 \mathrm{MHz}, \mathrm{CDCl}_{3}\right) \delta: 25.6(\mathrm{t}), 26.0(\mathrm{t})$, $26.9(\mathrm{t}), 30.1(\mathrm{t}), 33.5(\mathrm{t}), 45.9(\mathrm{~d}), 49.1(\mathrm{t}), 54.7(\mathrm{t}), 58.4(\mathrm{t}), 65.3(\mathrm{~d}), 126.6(\mathrm{~d}), 127.1(\mathrm{~d}), 127.4(\mathrm{~d})$, 127.9 (d), 128.3 (d), 128.4 (d), 130.3 (d), 132.5 (s) 133.0 (s), 133.6 (s), 134.9 (d), 135.0 (d), 140.9 (s). MS: $m / z 723\left(\mathrm{M}^{+}\right)$. Anal. calcd for $\mathrm{C}_{34} \mathrm{H}_{37} \mathrm{INPPd}$ : C, 56.41; H, 5.15; N, 1.93. Found C, 56.16; H, 5.29; $\mathrm{N}, 2.20$.

Crystallography: The crystallographic measurements were performed using an Enraf-Nonius CAD4 automated diffractometer, equipped with graphite-monochromated Mo-K $\alpha$ radiation $(\lambda=0.71073 \AA)$. Generator settings: $50 \mathrm{kV}, 30 \mathrm{~mA}$. A specimen of suitable size and quality was selected and glued on the tip of a glass fiber. The structures were solved by direct methods (SHELX86), which successfully located most of the non-hydrogen atoms. Subsequent refinement on $F^{2}$ using the SHELX97 as implemented in the WINGX suite of programs allowed location of the remaining non-hydrogen atoms. These were eventually inserted as atoms riding on the pertinent carbon atoms. Crystal data, details of the refinement procedure and final agreement factors, together with the full list of fractional atomic coordinates can be found in the pertinent CIF file. CCDC 613934. 


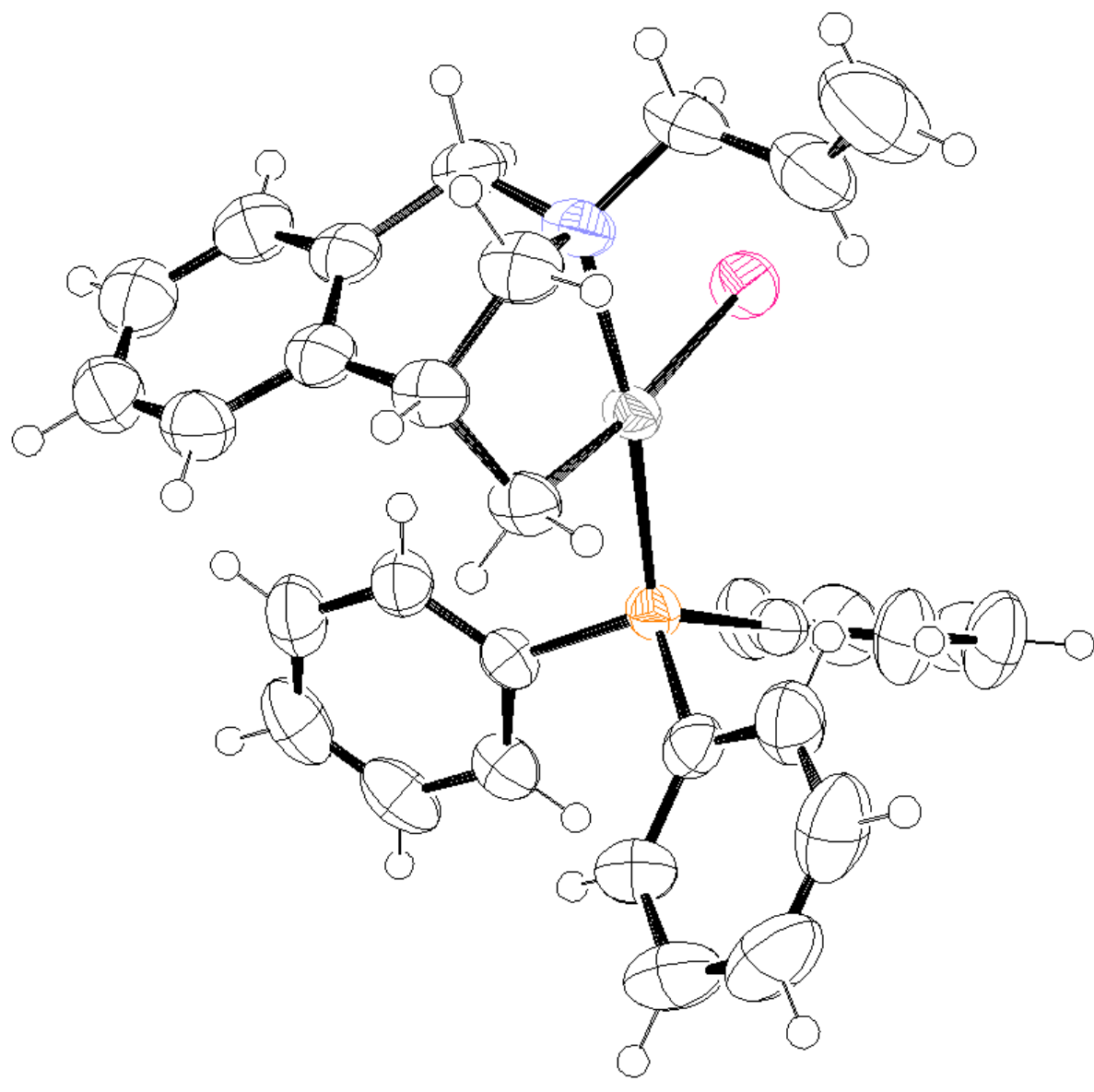

ORTEP drawing of compound 9b. Thermal ellipsoids are drawn at the 50\% probability level. Hydrogen atoms are given with arbitrary radii.

Crystal data: Triclinic, space group P-1 (No. 2), $a=9.458(2) ; b=11.332(2) ; c=13.604(2) \AA$ A $\alpha=$ 76.51(1); $\beta=86.68(2) ; \gamma=82.32(2)^{\circ} ; \mathrm{V}=1404.6(4) \AA ̊, ~ Z=2$. 
${ }^{1} \mathrm{H}-\mathrm{NMR}$ spectrum of compound $\mathbf{4 a}$.

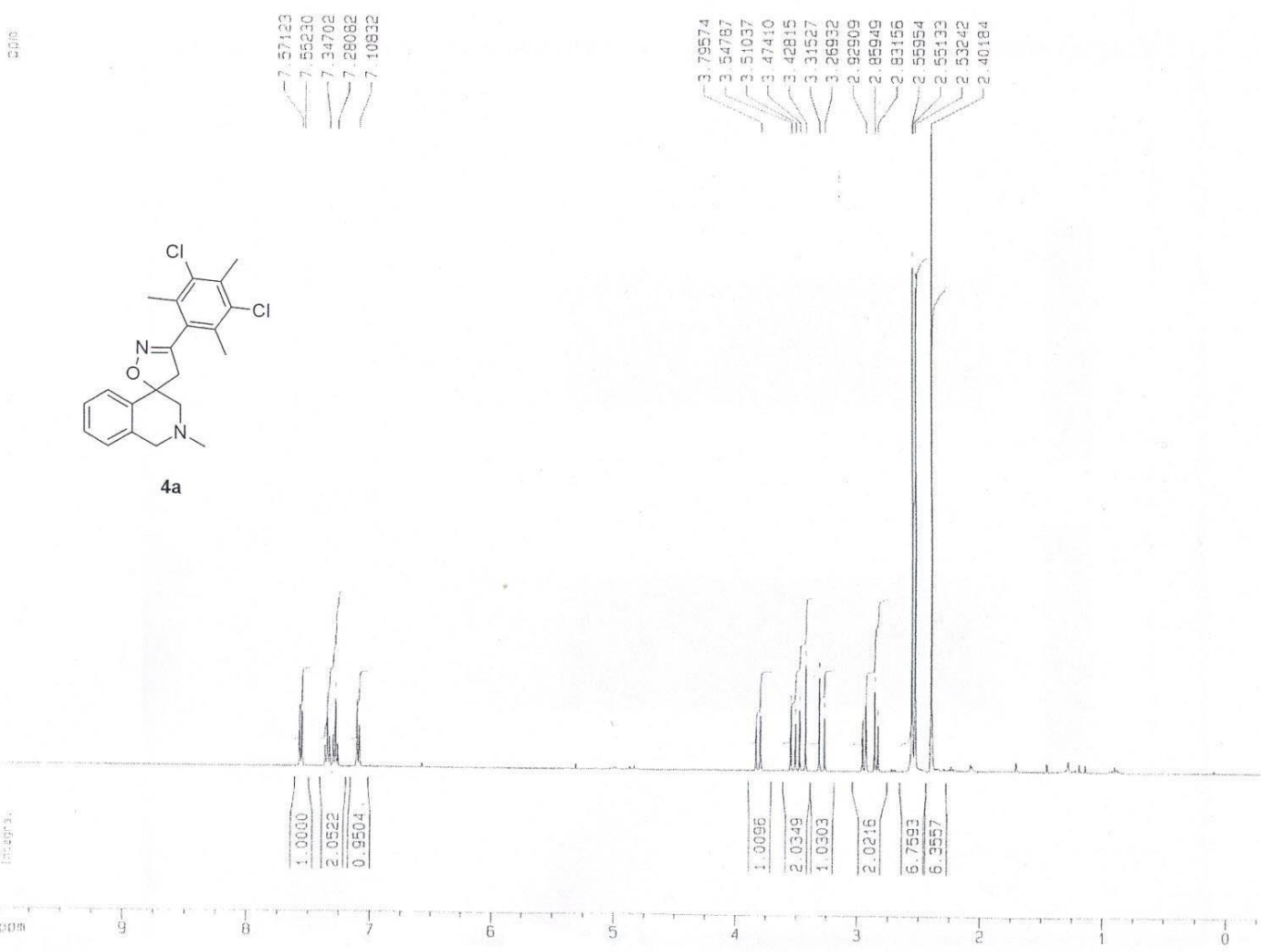

${ }^{13} \mathrm{C}-\mathrm{NMR}$ spectrum of compound $\mathbf{4 a}$.

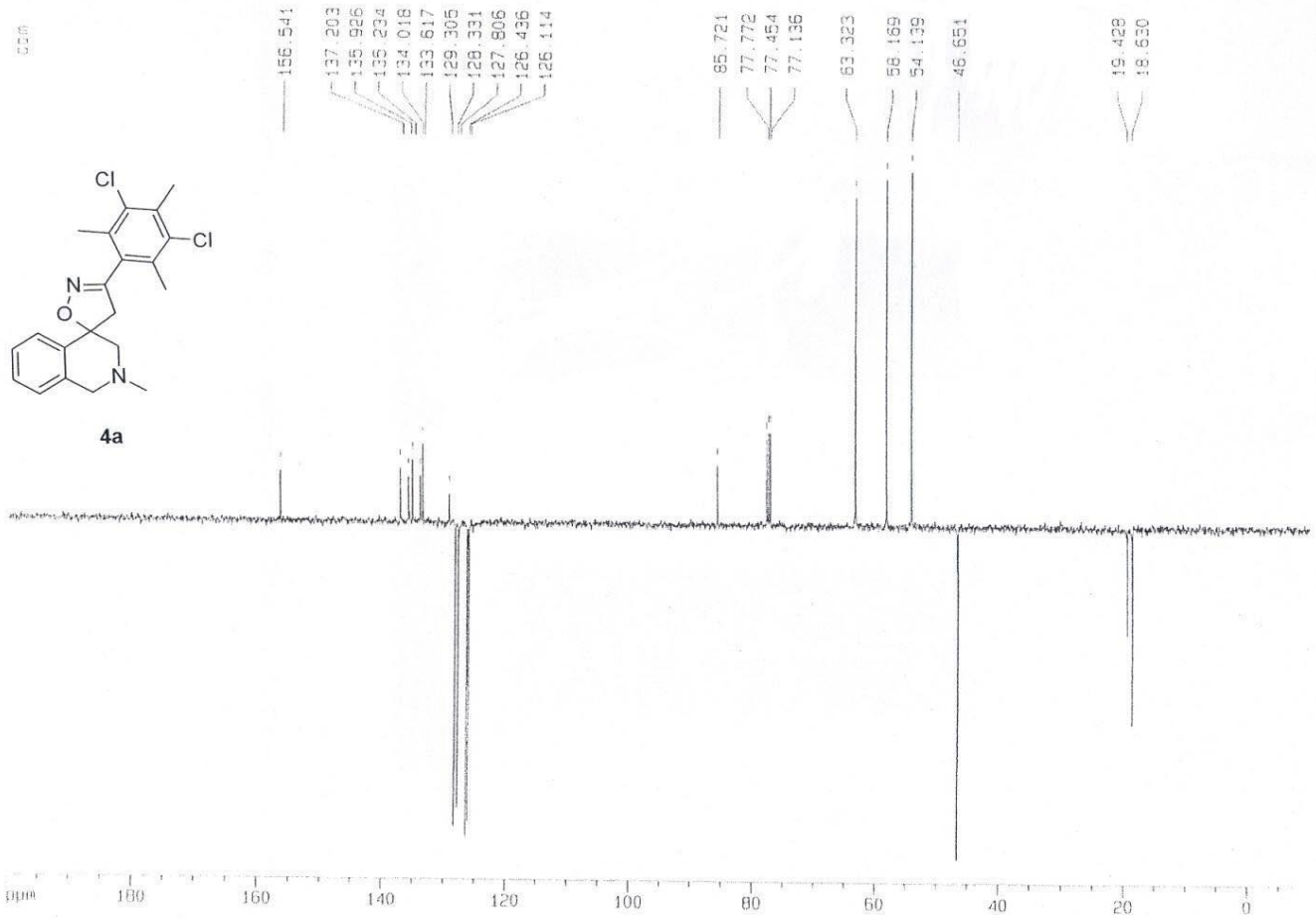


${ }^{1} \mathrm{H}-\mathrm{NMR}$ spectrum of compound $\mathbf{4 b}$.

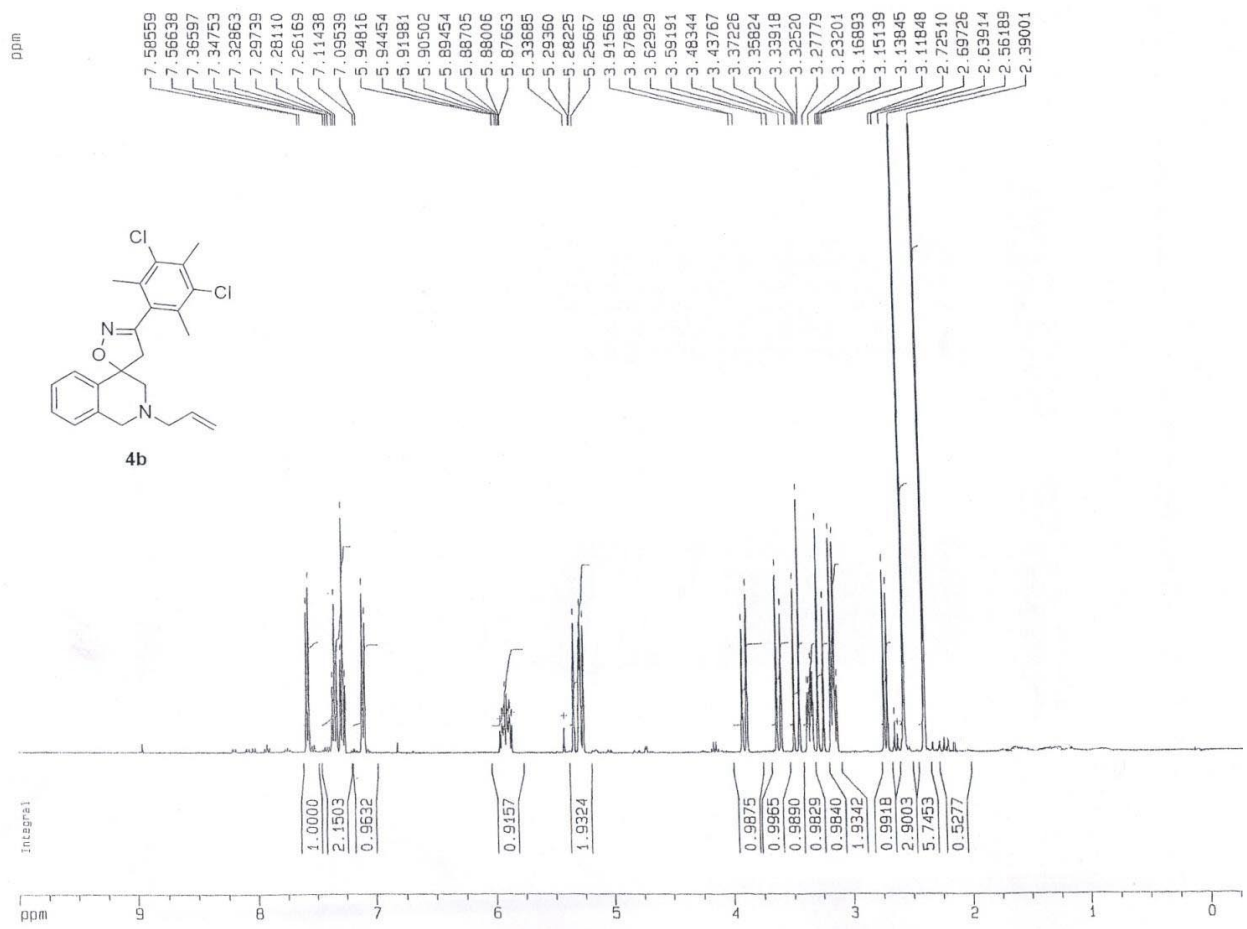

${ }^{13} \mathrm{C}-\mathrm{NMR}$ spectrum of compound $\mathbf{4 b}$.
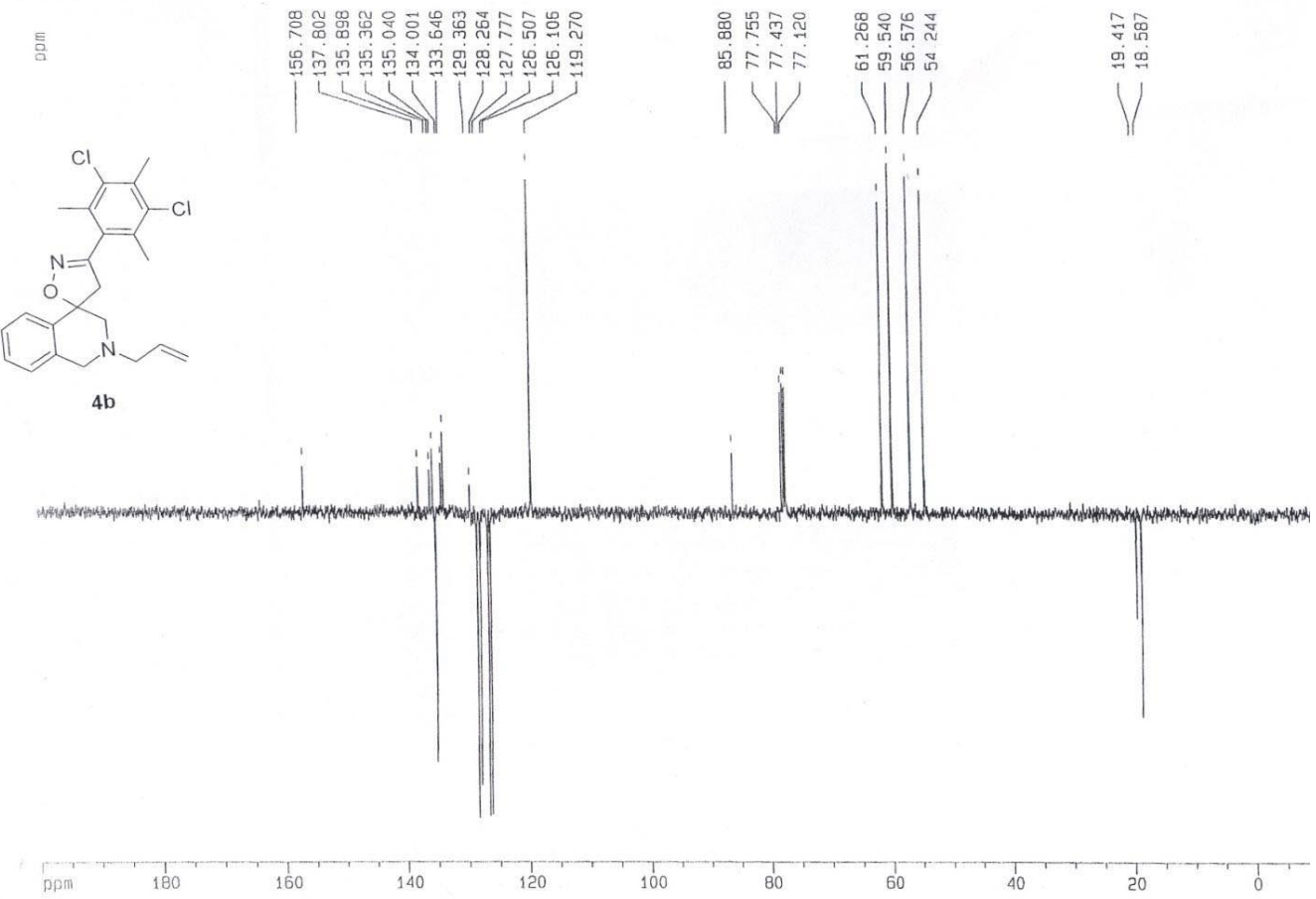
${ }^{1} \mathrm{H}-\mathrm{NMR}$ spectrum of compound $\mathbf{4 c}$.

言
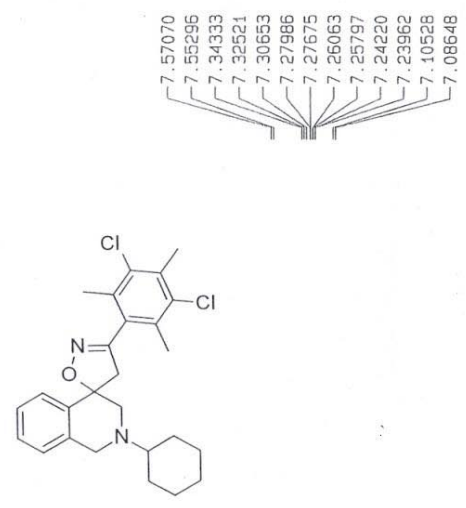

4c
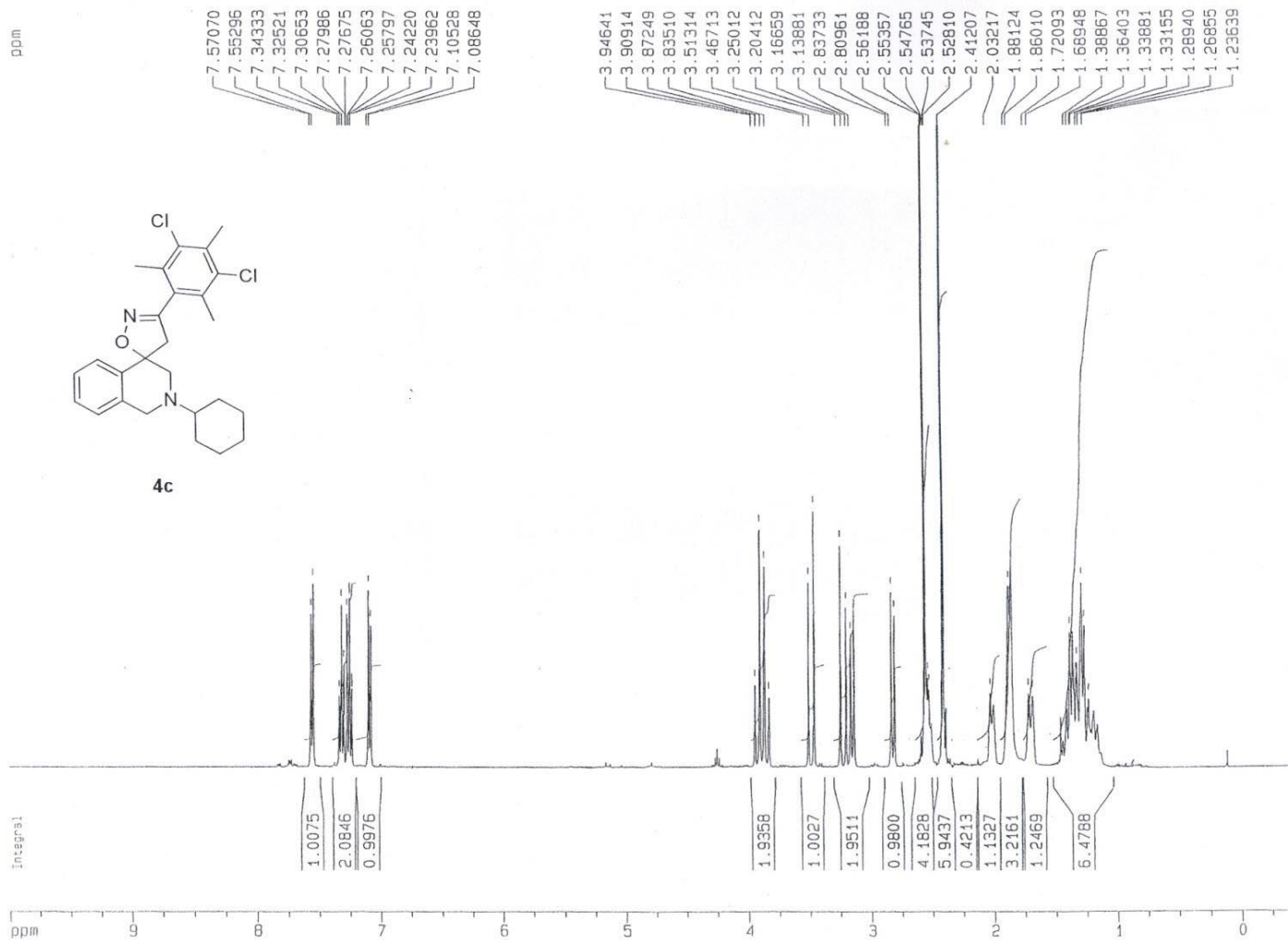

${ }^{13} \mathrm{C}-\mathrm{NMR}$ spectrum of compound $\mathbf{4 c}$.

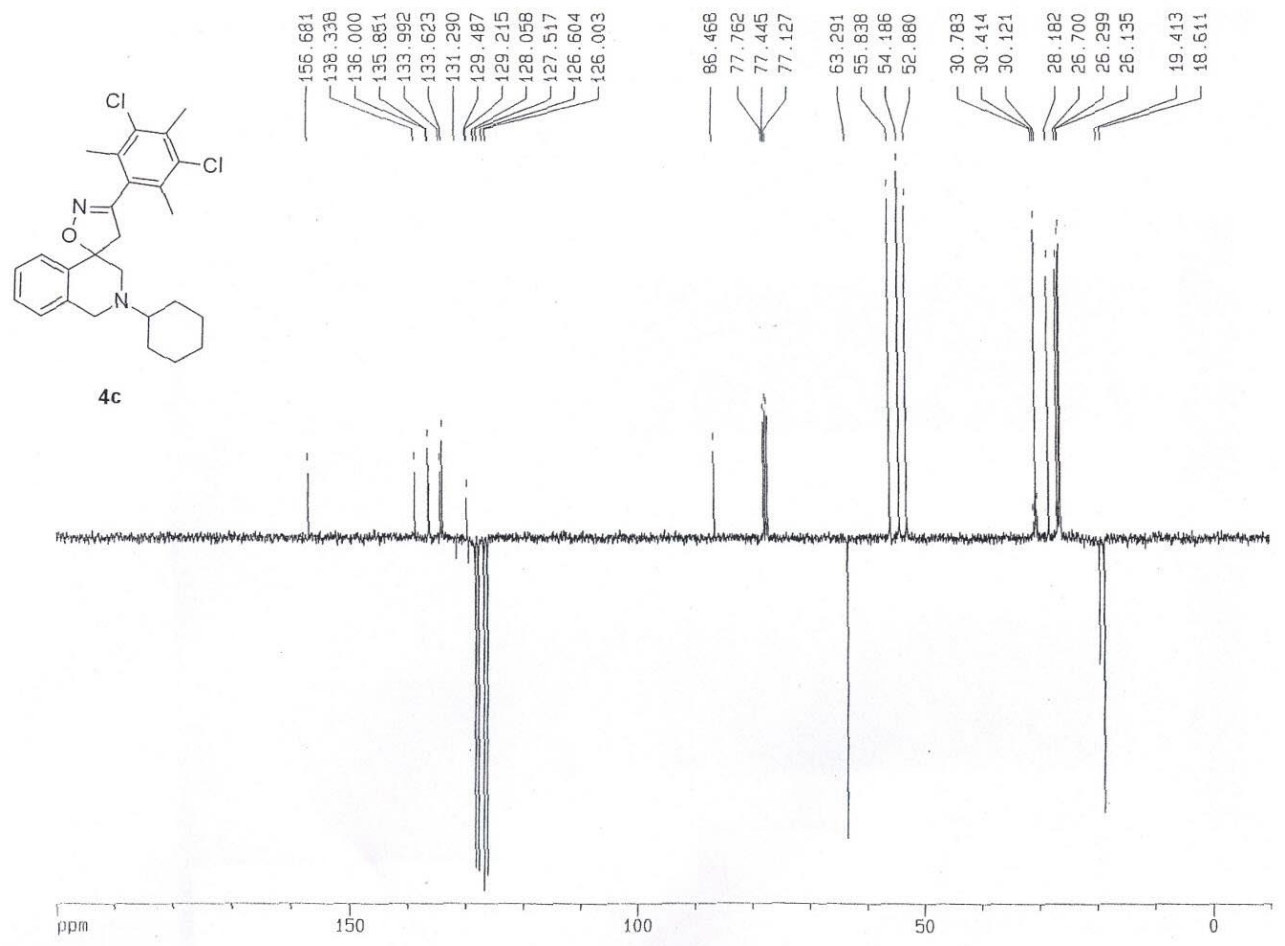


${ }^{1} \mathrm{H}-\mathrm{NMR}$ spectrum of compound $\mathbf{4 d}$.

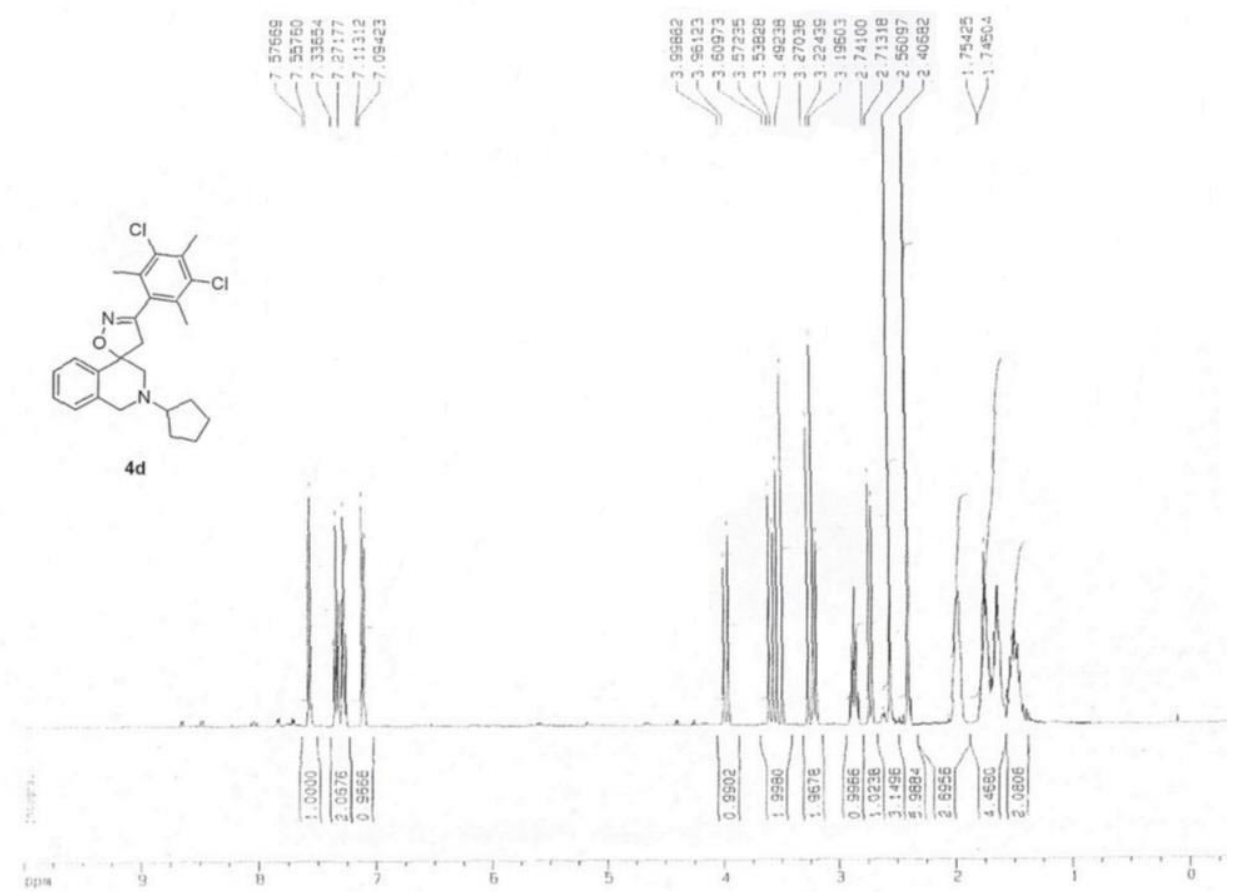

${ }^{13} \mathrm{C}-\mathrm{NMR}$ spectrum of compound $\mathbf{4 d}$.

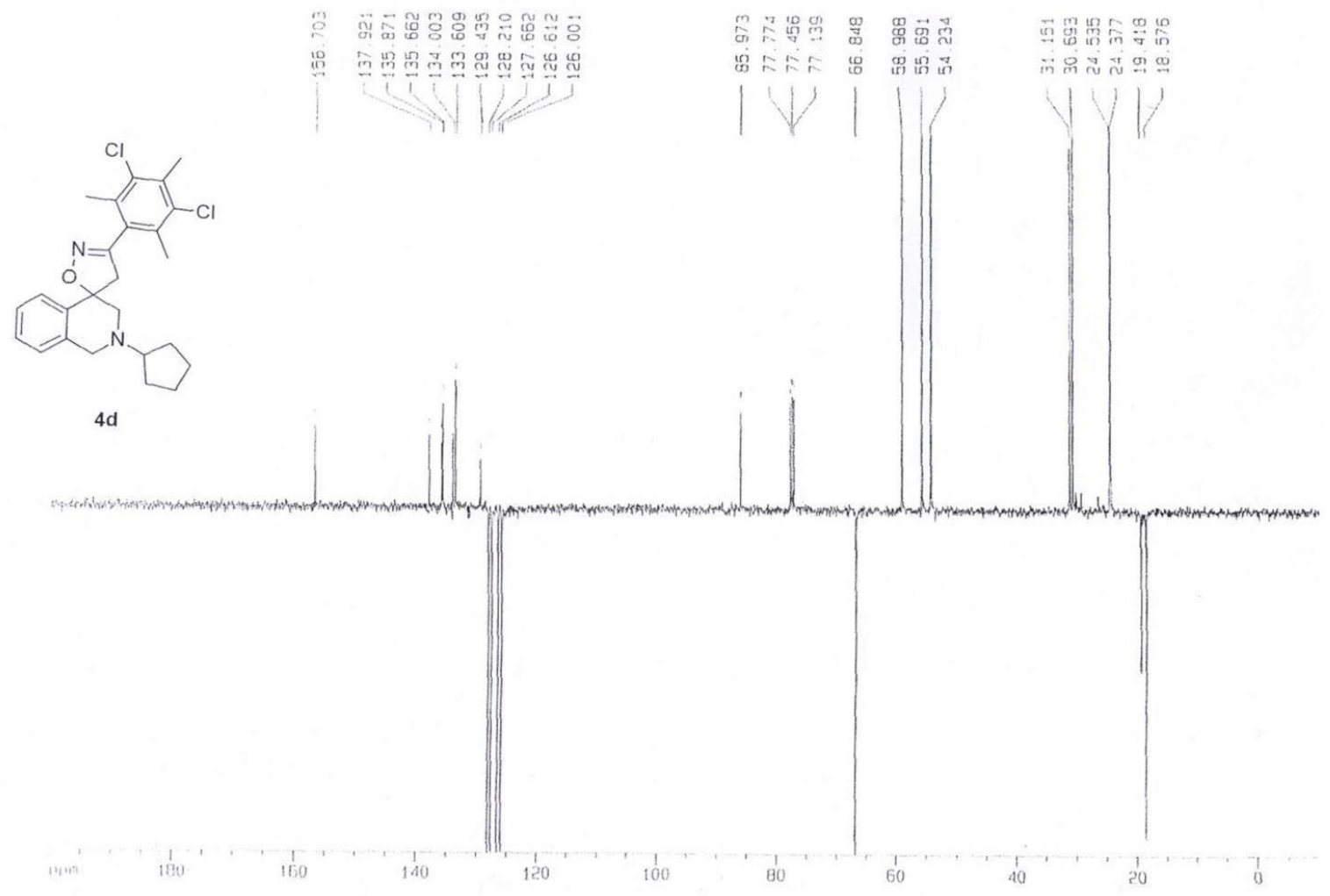


${ }^{1} \mathrm{H}-\mathrm{NMR}$ spectrum of compound $\mathbf{6 a}$.

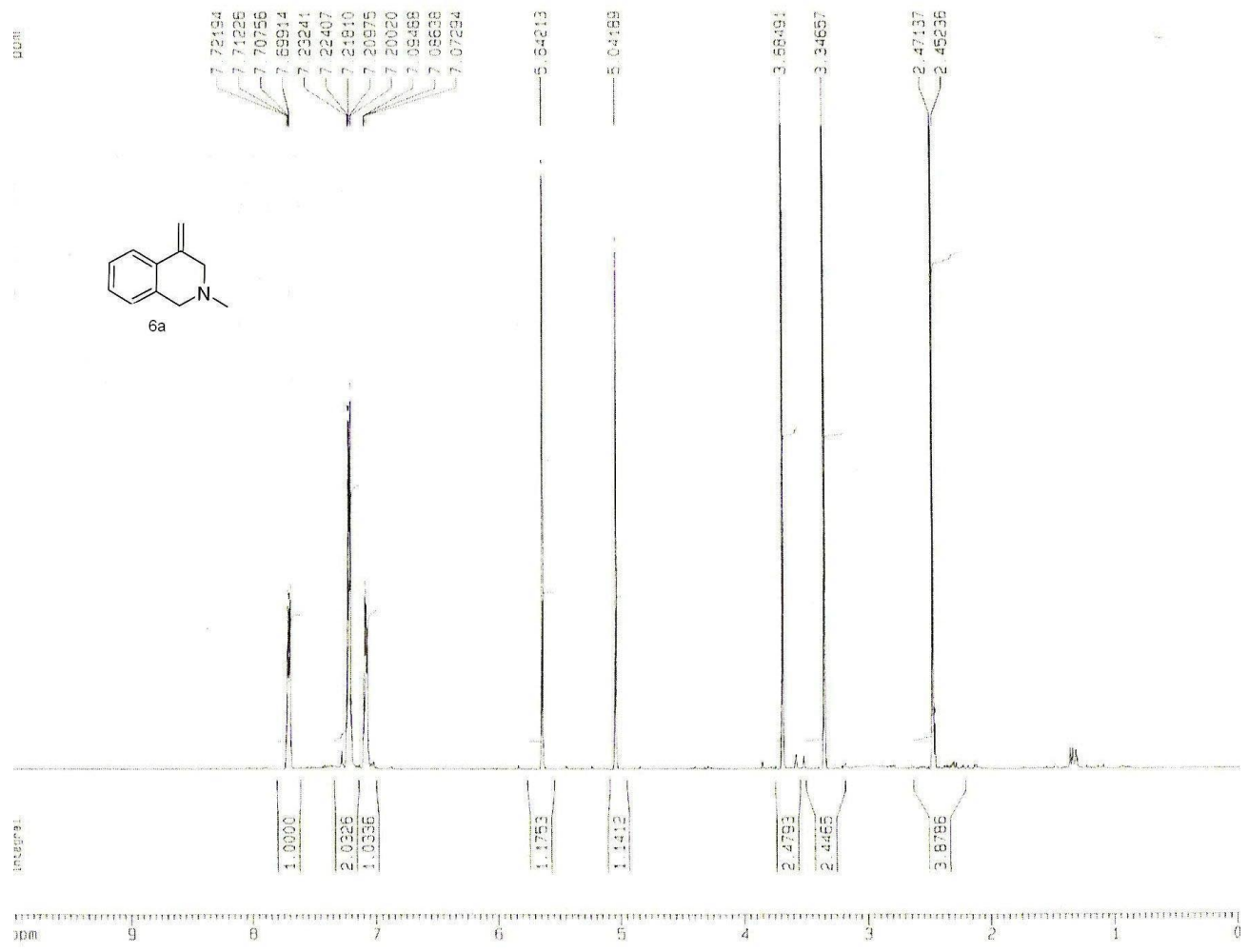

${ }^{13} \mathrm{C}-\mathrm{NMR}$ spectrum of compound $\mathbf{6 a}$.

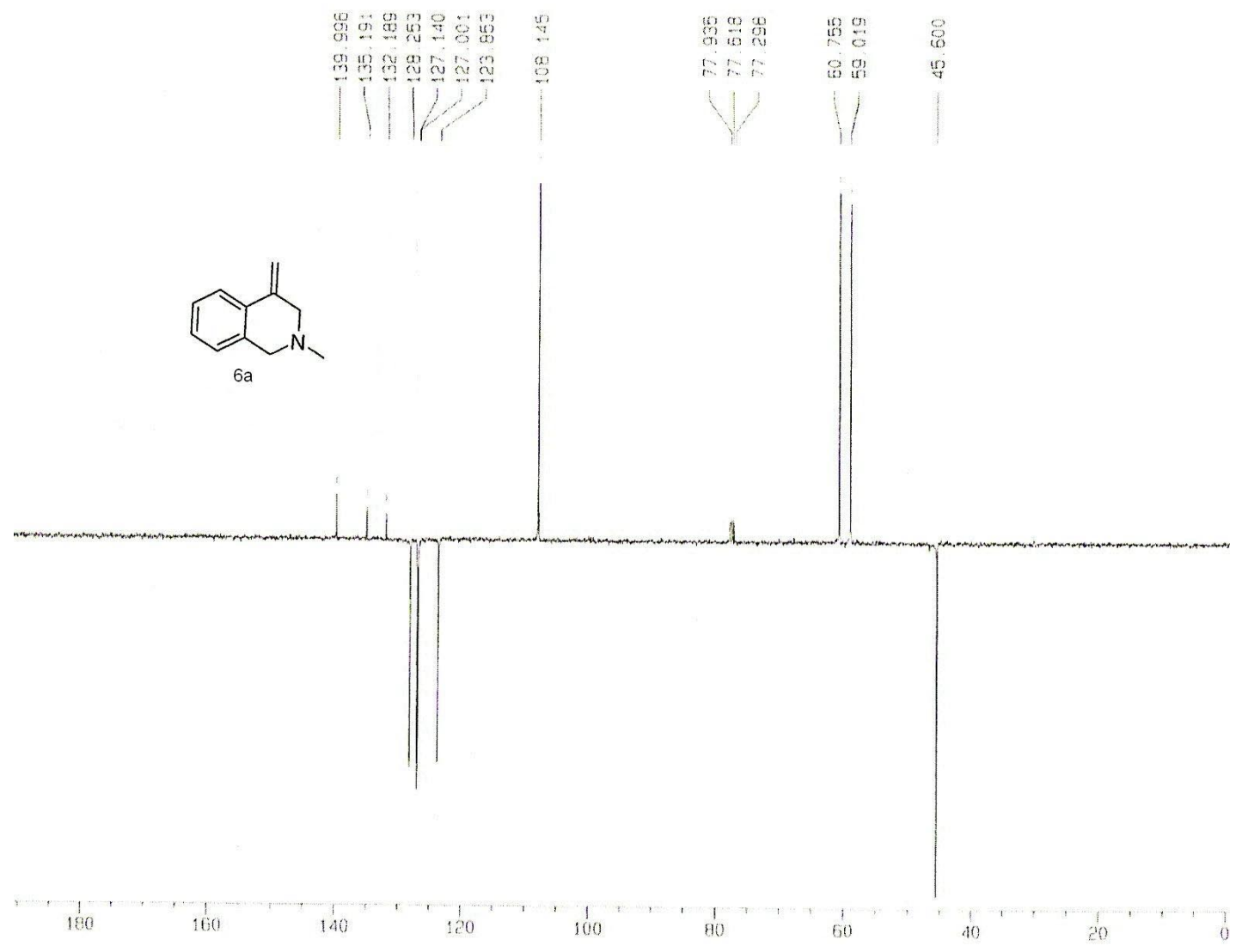


${ }^{1} \mathrm{H}-\mathrm{NMR}$ spectrum of compound $\mathbf{6} \mathbf{b}$.
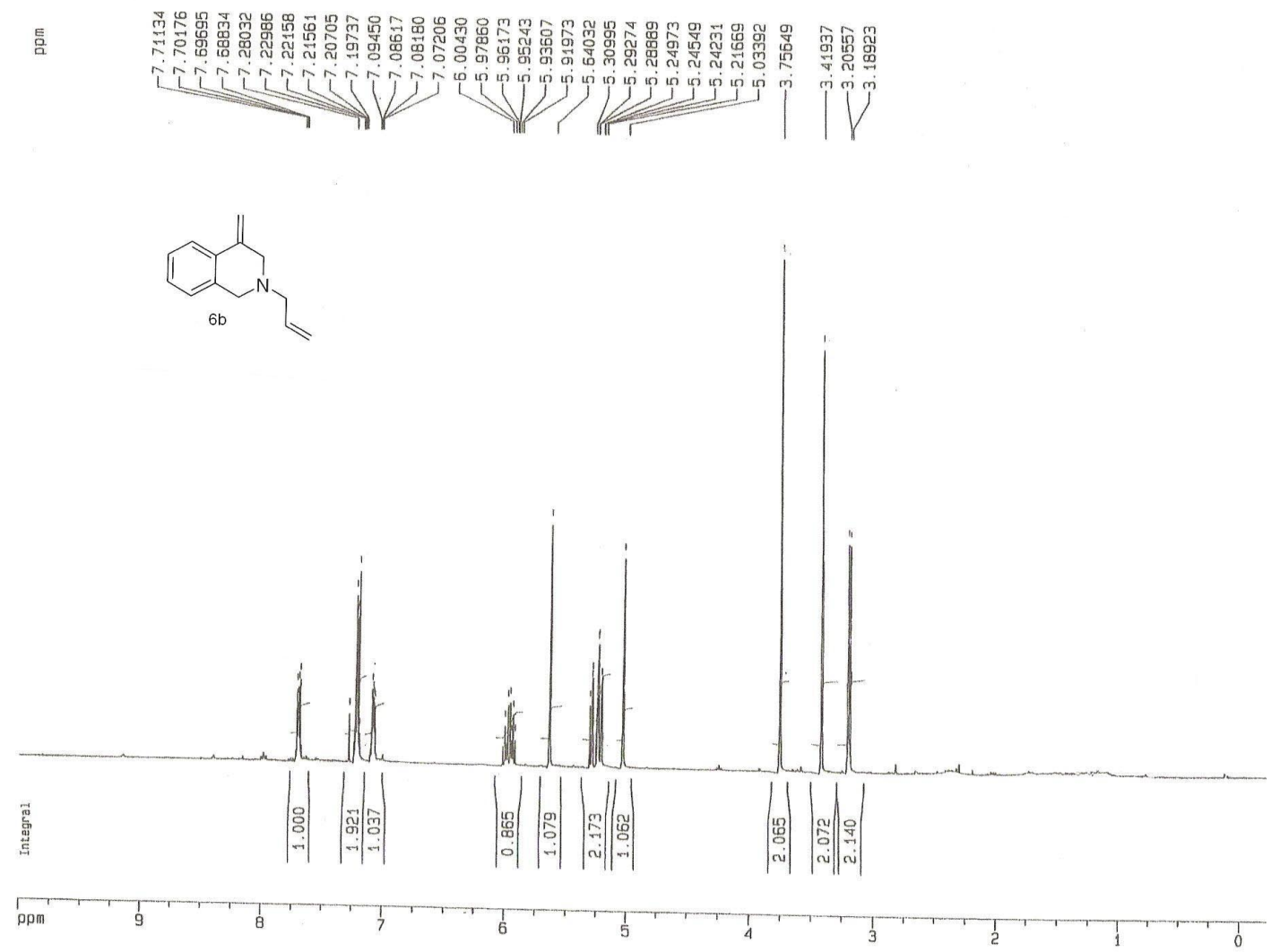

${ }^{13} \mathrm{C}$-NMR spectrum of compound $\mathbf{6 b}$.

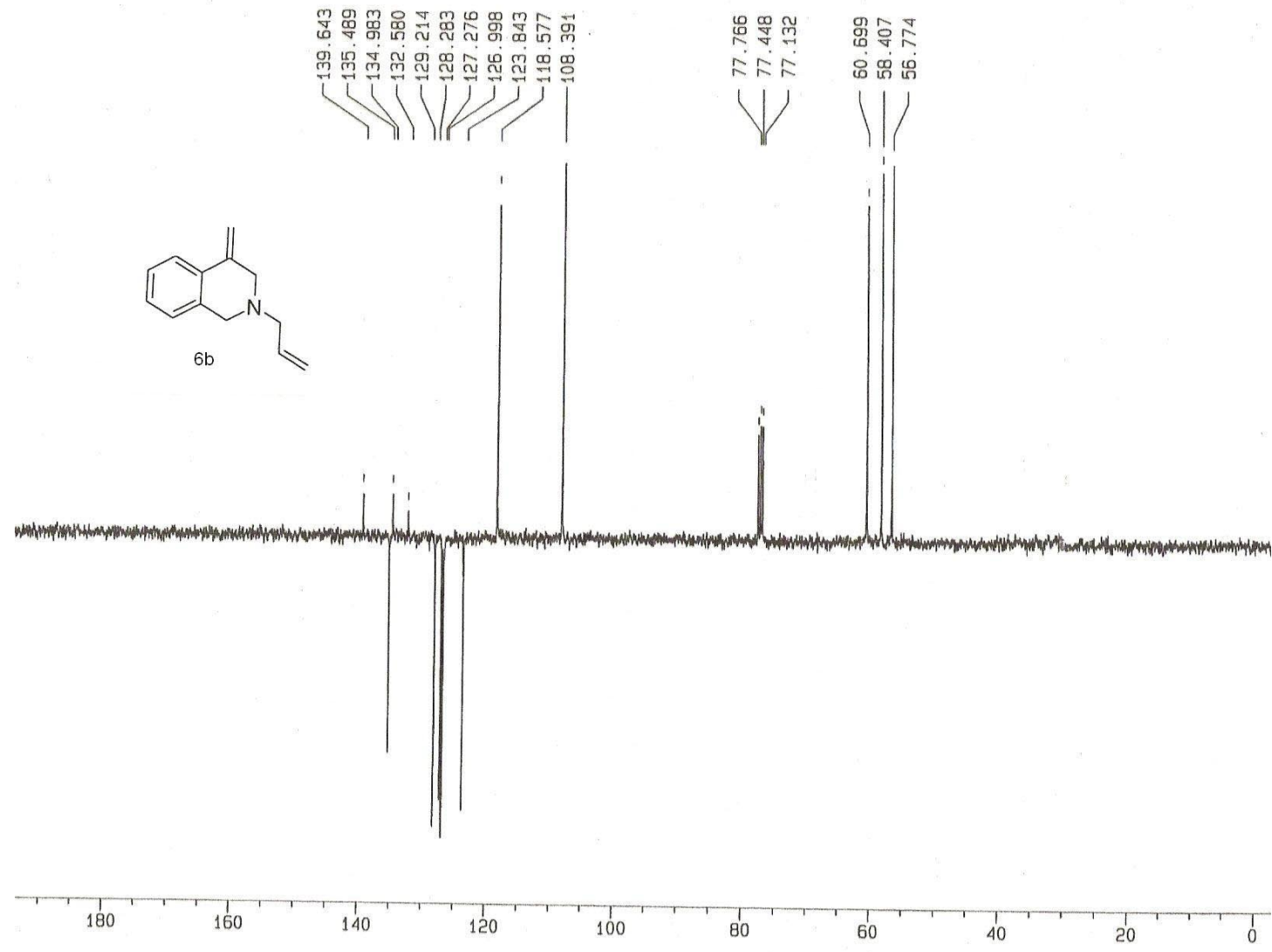


${ }^{1} \mathrm{H}-\mathrm{NMR}$ spectrum of compound $\mathbf{6 c}$.

镸
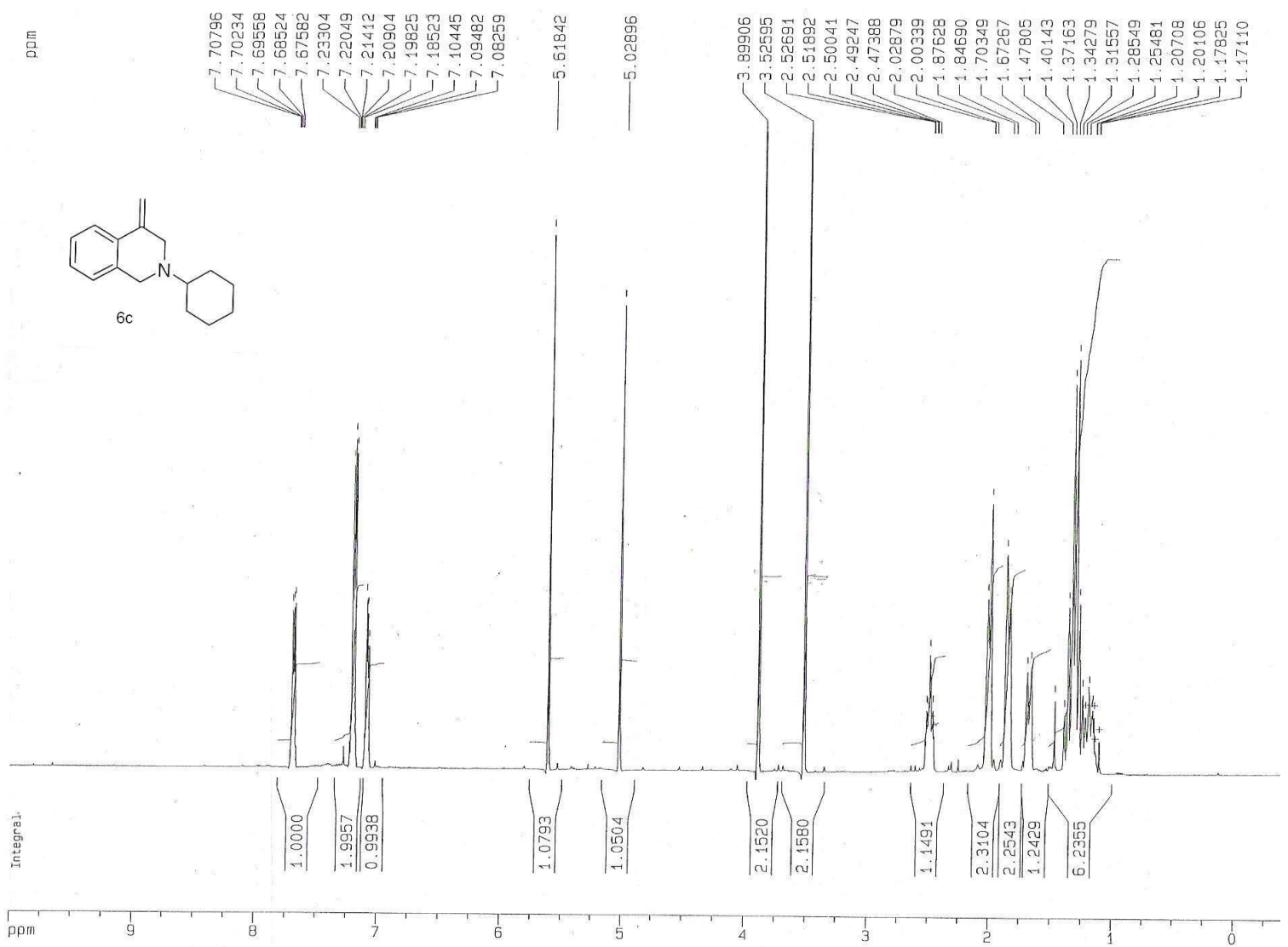

\section{${ }^{13} \mathrm{C}-\mathrm{NMR}$ spectrum of compound $\mathbf{6 c}$.}
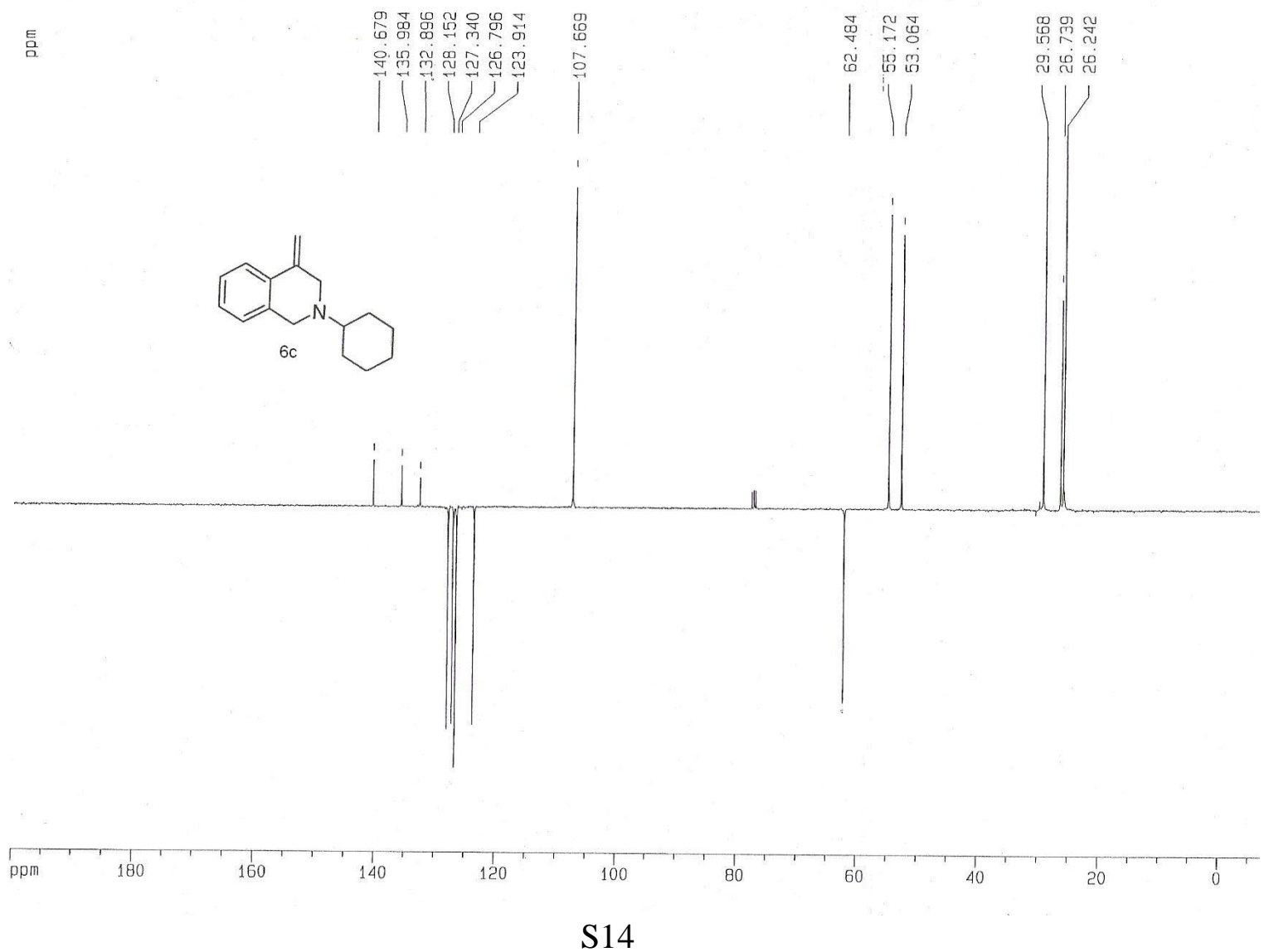
${ }^{1} \mathrm{H}-\mathrm{NMR}$ spectrum of compound $\mathbf{9 a}$.

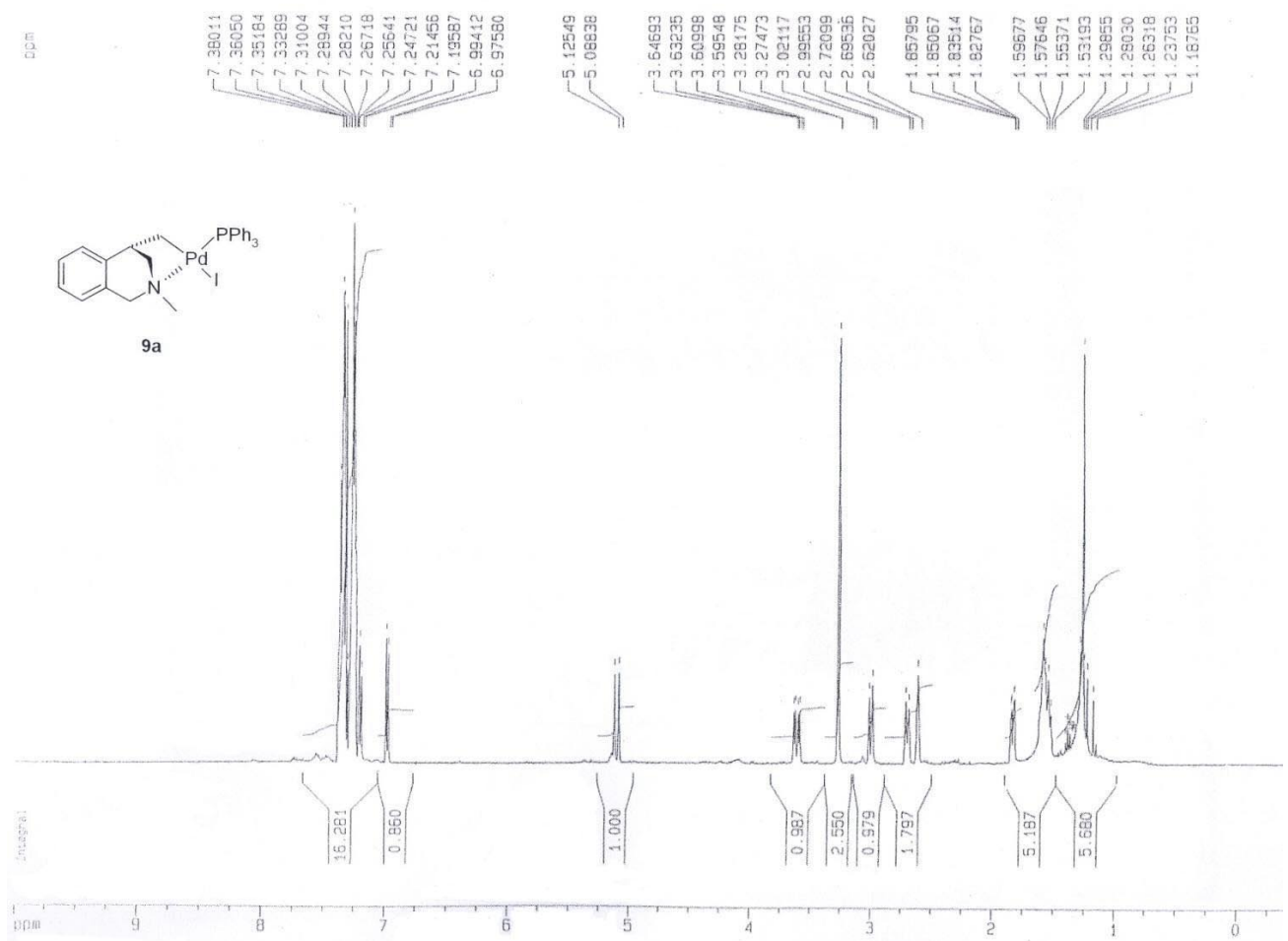

${ }^{13} \mathrm{C}-\mathrm{NMR}$ spectrum of compound $\mathbf{9 a}$.

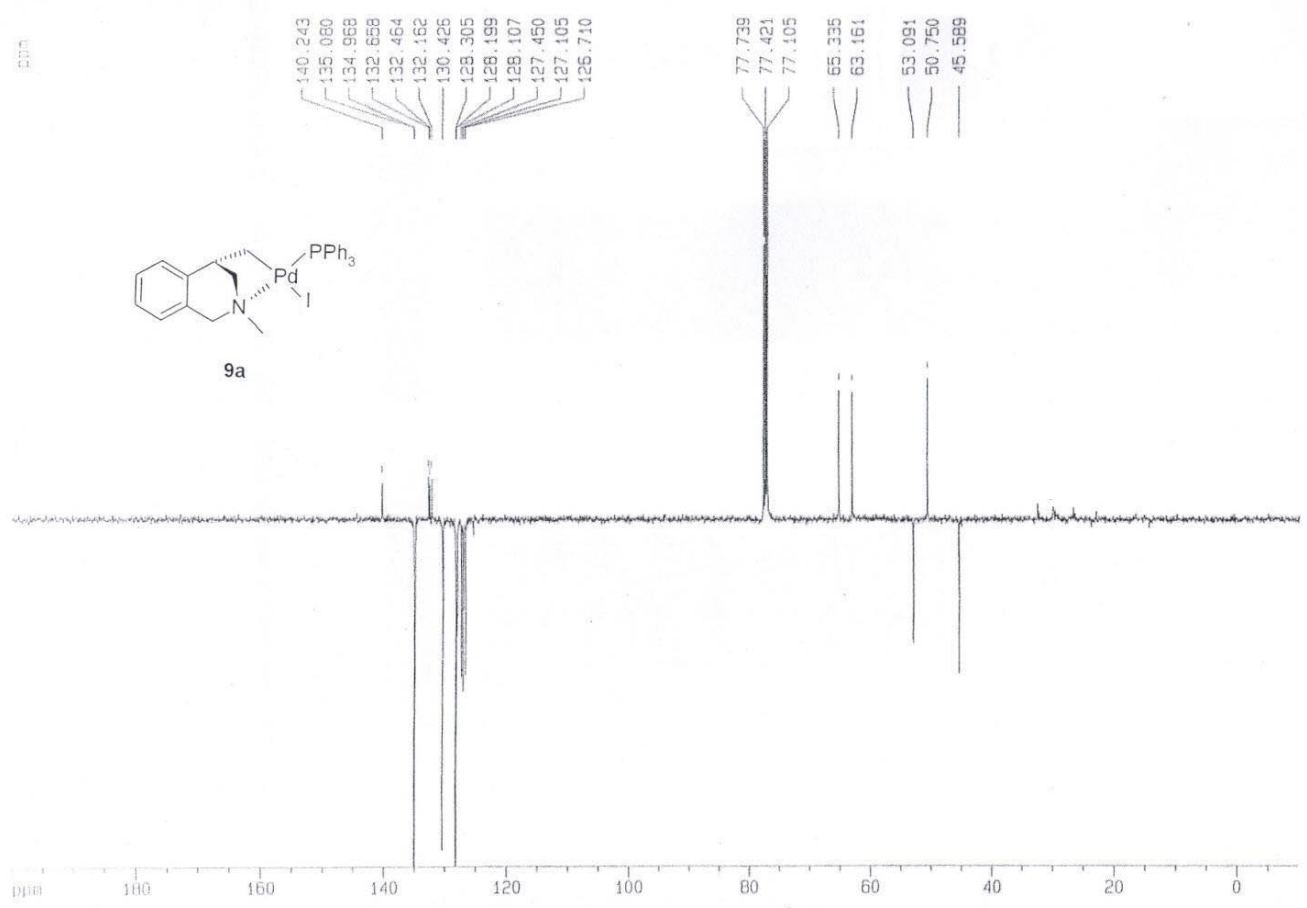


${ }^{1} \mathrm{H}-\mathrm{NMR}$ spectrum of compound $\mathbf{9 b}$.

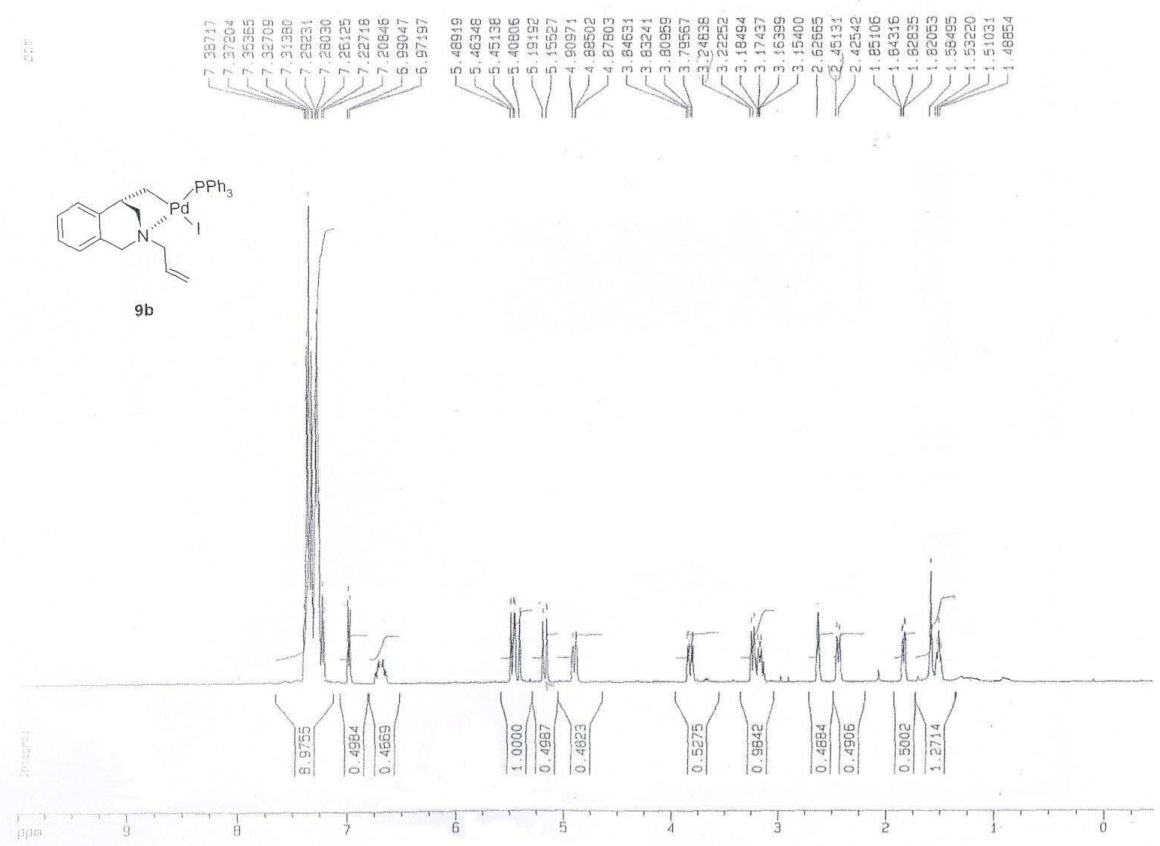

${ }^{13} \mathrm{C}-\mathrm{NMR}$ spectrum of compound $\mathbf{9 b}$.

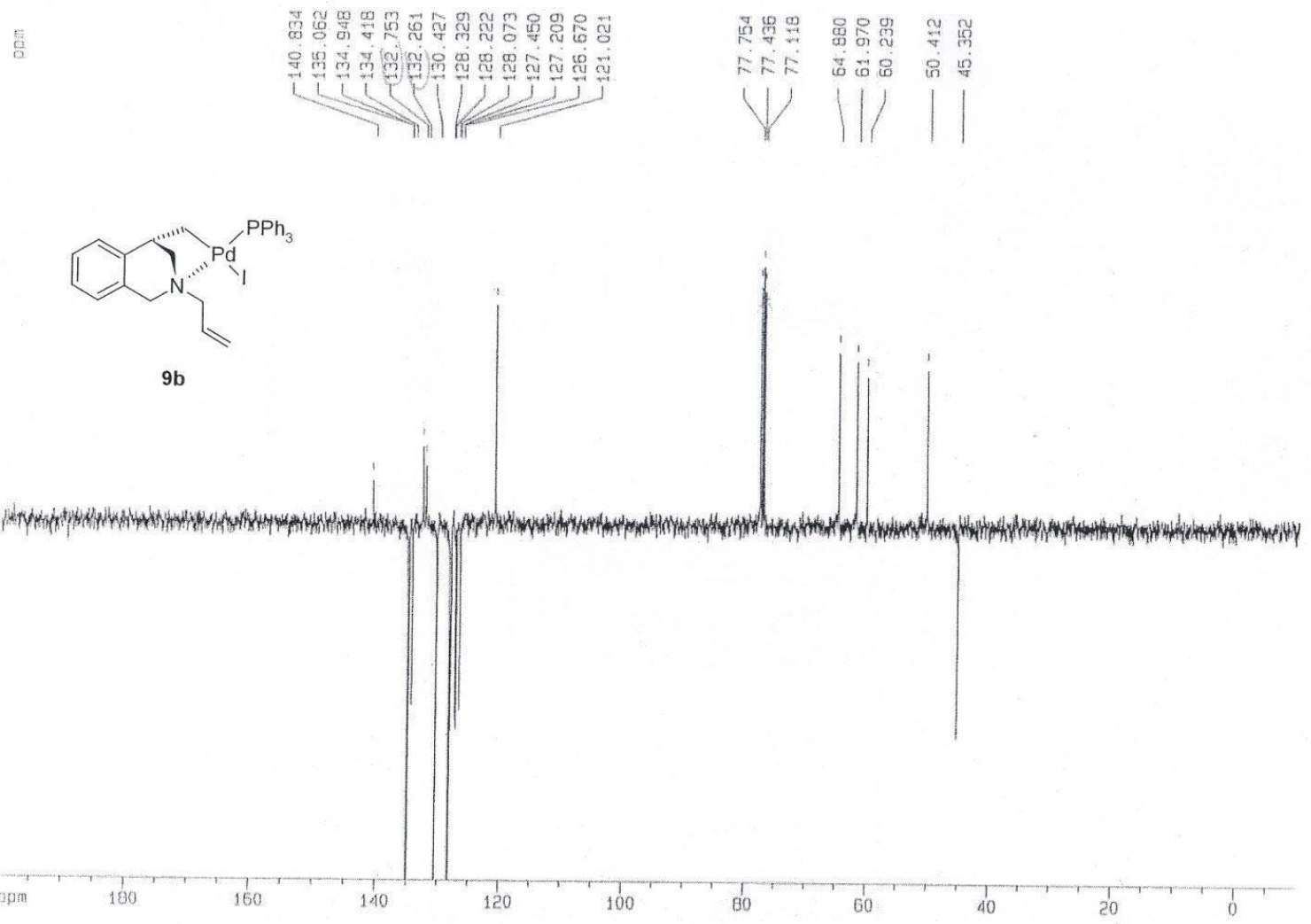


${ }^{1} \mathrm{H}-\mathrm{NMR}$ spectrum of compound $\mathbf{9 c}$.
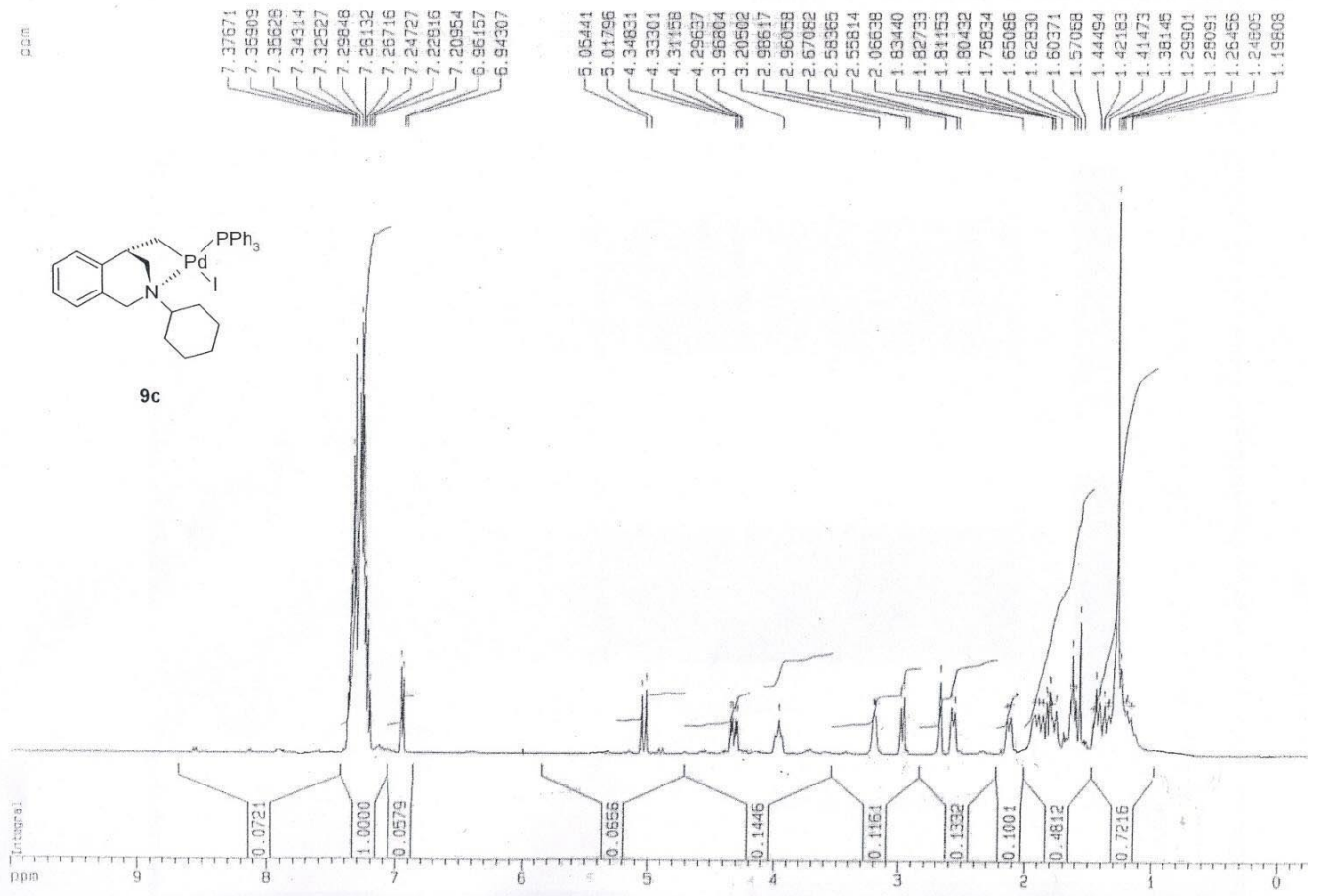

${ }^{13} \mathrm{C}-\mathrm{NMR}$ spectrum of compound 9c.

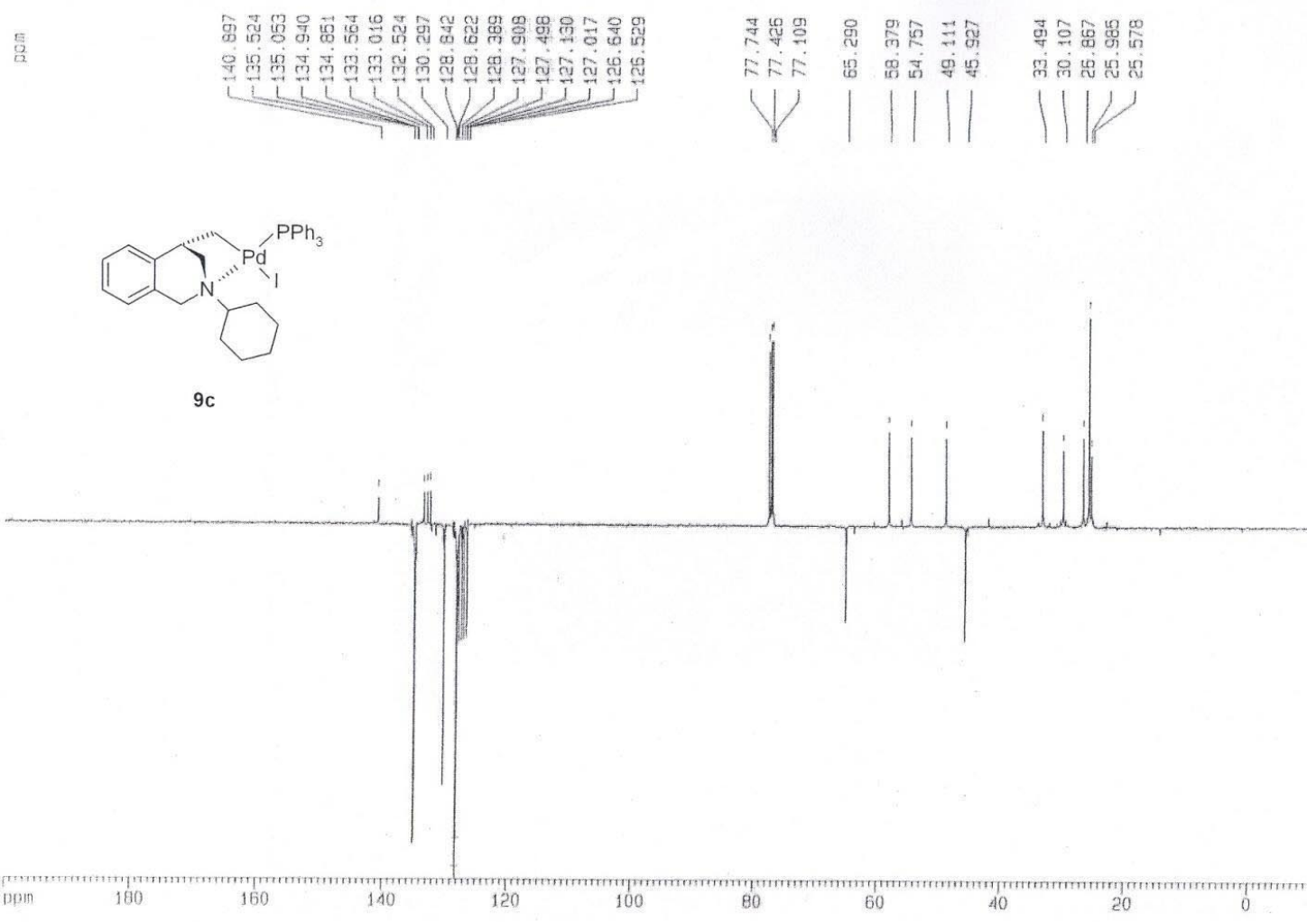

